

\section{Argonne National Laboratory}

Argonne National Laboratory, with facilities in the states of Illino is and Idaho, is owned by the United States government, and operated by the University

of Chicago under the provisions of a contract with the Department of Energy.

This technical report is a product of Argonne's Energy Systems Division.

For information on the division's scientific and engineering activities, contact:

Director, Energy Systems Division

Argonne National Laboratory

Argonne, Illinois 60439-4815

Telephone (708) 972-3724

\section{Disclaimer}

This report was prepared as an account of work sponsored by an agency of the United States Government. Neither the United States Government nor any agency thereof, nor any of their employees, makes any warranty, express or implied, or assumes any legal liability or responsibility for the accuracy, completeness, or usefulness of any information, apparatus, product, or process disclosed, or represents that its use would not infringe privately owned rights. Reference herein to any specific commercial product, process, or service by trade name, trademark, manufacturer, or otherwise, does not necessarily constitute or imply its endorsement, recommendation, or favoring by the United States Government or any agency thereof. The views and opinions of authors expressed herein do not necessarily state or reflect those of the United States Government or any agency thereof.

Reproduced from the best available copy. Available from the National Technical Information Service (NTIS), U.S. Department of Commerce, 5285 Port Royal Road, Springfield, Virginia 22161.

NTIS price codes:

Printed copy $A 03$

Microfiche A01 
ANLJEAIS-3

\section{RESRAD Parameter Sensitivity Analysis}

by J.-J. Cheng, C. Yu, and A.J. Zielen

Environmental Assessment and Information Sciences Division,

Argonne National Laboratory, 9700 South Cass Avenue, Argonne, Illinois 60439

August 1991

Work sponsored by United States Department of Energy,

Office of Environmental Restoration and Waste Management 


\section{CONTENTS}

ACKNOWLEDGMENTS $\ldots \ldots \ldots \ldots \ldots \ldots \ldots \ldots \ldots \ldots \ldots \ldots \ldots$ vi

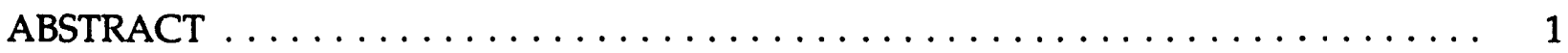

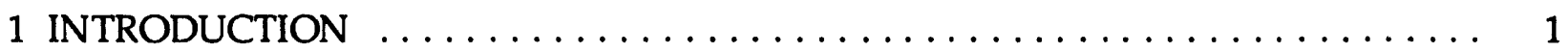

2 SENSITIVITY ANALYSIS VIA THE GRESS SOFTWARE PACKAGE $\ldots \ldots \ldots \ldots$

2.1 Modification of RESRAD for GRESS Precompilation $\ldots \ldots \ldots \ldots \ldots \ldots \ldots$

2.2 GRESS Enhancement Sensitivity Analysis Results $\ldots \ldots \ldots \ldots$

3 SENSITIVITY ANALYSIS VIA DIRECT PARAMETER PERTURBATION $\ldots \ldots \ldots$

3.1 Perturbation of Input Parameters $\ldots \ldots \ldots \ldots \ldots \ldots \ldots \ldots \ldots \ldots \ldots$

3.2 Calculational Procedure . . . . . . . . . . . . . . . . . . . . 14

3.3 Comparison of Direct Parameter Perturbation Results with GRESS

Enhancement Results ......................... 16

4 SENSITIVITY ANALYSIS VIA RESRAD'S GRAPHICS CAPABILITY $\ldots \ldots \ldots \ldots$

4.1 Invoking Sensitivity Analysis from the RESRAD Input Screen . . . . . . . . 17

4.2 Viewing the Sensitivity Analysis Results . . . . . . . . . . . . . . . . . 19

4.3 Comparing Graphic Results with GRESS Enhancement and Direct Parameter Perturbation Results . . . . . . . . . . . . . 20

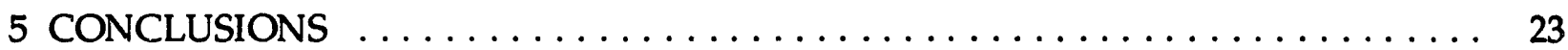

REFERENCES $\ldots \ldots \ldots \ldots \ldots \ldots \ldots \ldots \ldots \ldots \ldots \ldots \ldots \ldots \ldots \ldots \ldots$

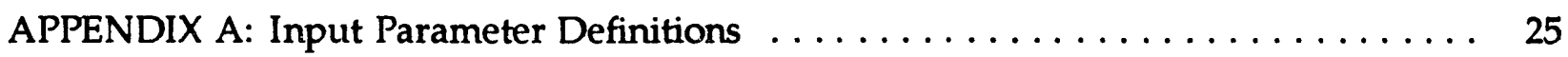

APPENDIX B: RESRAD Parameters Eligible for Sensitivity Analysis $\ldots \ldots \ldots \ldots \ldots$

APPÉNDIX C: RESRAD Sensitivity Input Data File . . . . . . . . . . . . . . 29

APPENDIX D: Typical Results of GREGS Sensitivity Analysis $\ldots \ldots \ldots \ldots$

APPENDIX E: RESRAD/VAX Version Summary Report . . . . . . . . . . . 31 


\section{FIGURES}

1 Schematic Representation of RESRAD Pathways $\ldots \ldots \ldots \ldots \ldots \ldots \ldots$

2 Flow Diagram of Direct Parameter Perturbation Method $\ldots \ldots \ldots \ldots \ldots$

3 Dose/Source Ratio of Water-Independent and Water-Dependent Pathway

Subtotals of Uranium $-234 \ldots \ldots \ldots \ldots \ldots \ldots \ldots \ldots \ldots$

4 Dose/Source Ratio of Water-Dependent Pathways of Uranium-238 $\ldots \ldots \ldots$

5 Input Form R013 for Cover and Contaminated Zone Hydrological Data . . . . . 18

6 Input Form R013 with Sensitivity Analysis Pop-Up Menu . . . . . . . . . . . . 19

7 Total Dose of All Isotopes Summed over All Pathways with

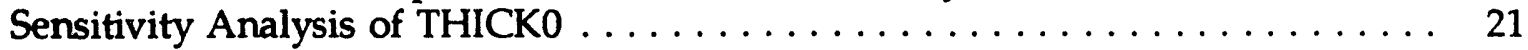

8 Total Dose of All Isotopes Summed over All Pathways with

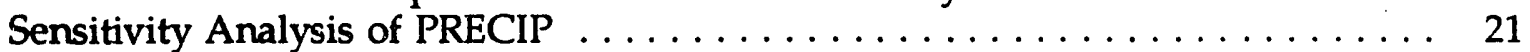

9 Total Dose of All Isotopes Summed over All Pathways with

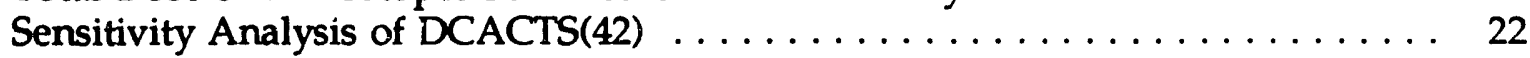

C.1 SENSE.DAT File $\ldots \ldots \ldots \ldots \ldots \ldots \ldots \ldots \ldots \ldots \ldots \ldots \ldots \ldots$

D.1 GRESS Sensitivity Analysis Results for TDOSE( 8$) \ldots \ldots \ldots \ldots \ldots \ldots$

\section{TABLES}

1 Sensitivity Analysis of Total Dose at 100 Years $\ldots \ldots \ldots \ldots$

2 Sensitivity Analysis of Total Dose at 300 Years $\ldots \ldots \ldots \ldots \ldots$

3 Sensitivity Analysis of Total Dose at 1000 Years $\ldots \ldots \ldots \ldots$

4 Sensitivity Analysis of Total Dose at 3000 Years $\ldots \ldots \ldots \ldots$

5 Sensitivity Analysis of Dose/Source Ratio from Uranium-234 in the Water-Independent Plant Pathway at 30 Years . . . . . . . . . . . . . 10

6 Sensitivity Analysis of Dose/Source Ratio from Uranium-234 in the Ground Pathway at 300 Years . . . . . . . . . . . . . . . . . 11 


\section{TABLES (Cont'd)}

7 Sensitivity Analysis of Dose/Source Ratio from Uranium- 238

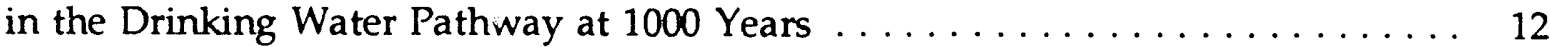

8 Sensitivity Analysis of Dose/Source Ratio from Uranium-238 in the Drinking Water Pathway at 3000 Years 


\section{ACKNOWLEDGMENTS}

The authors would like to thank Kenneth Evans, Jr. for his initial work in setting up the Gradient Enhanced Software System package; Andrew Wallo III and W. Alexander Williams of the U.S. Department of Energy and Donald Mackenzie of H \& R Technical Associates, Inc., for their review of this report; and Patricia E. Hollopeter for her editorial efforts. 


\title{
RESRAD PARAMETER SENSITIVITY ANALYSIS
}

\author{
by
}

\author{
J.-J. Cheng, C. Yu, and A.J. Zielen
}

\begin{abstract}
Three methods were used to perform a sensitivity analysis of RESRAD code input parameters -- enhancement of RESRAD by the Gradient Enhanced Software System (GRESS) package, direct parameter perturbation, and graphic comparison. Evaluation of these methods indicated that (1) the enhancement of RESRAD by GRESS has limitations and should be used cautiously, (2) direct parameter perturbation is tedious to implement, and (3) the graphics capability of RESRAD 4.0 is the most direct and convenient method for performing sensitivity analyses. This report describes procedures for implementing these methods and presents a comparison of results.
\end{abstract}

\section{INTRODUCTION}

A sensitivity analysis of the input parameters of RESRAD, a computer code for implementing U.S. Department of Energy (DOE) guidelines for residual radioactive material (Gilbert et al. 1989), was performed. The RESRAD code establishes soil cleanup criteria on the basis of predictions of radiation doses received by an individual from radionuclides contained in the soil and transported in the environment. The RESRAD code was used to consider seven environmental transport pathways: external radiation from contaminated soil materials; internal radiation from inhalation of contaminated dust particles; internal radiation from ingestion of plant foods grown on-site and irrigated with water drawn from an on-site well or pond; internal radiation from ingestion of meat from livestock fed with fodder grown on-site and water drawn from an on-site well or pond; internal radiation from ingestion of milk from livestock fed with fodder grown on-site and water drawn from an on-site well or pond; internal radiation from ingestion of aquatic food (fish) from a nearby pond; and internal radiation from drinking water from an on-site well or pond. Figure 1 is a schematic representation of the relationships among the pathways. As with any predictive model, the accuracy of the predictions depends on the accuracy of the input parameters (see Appendixes A and B). This report presents the results of a sensitivity analysis of RESRAD input parameters. Parameters with high sensitivities will have a greater effect on the predicted results and, therefore, these parameters should be determined more accurately. Accurate sensitivity analysis results can then be used to set priorities for gathering input data.

Three methods were used to perform a sensitivity analysis of RESRAD input parameters: (1) application of the Gradient Enhanced Software System (GRESS), a sensitivity analysis package developed at Oak Ridge National Laboratory (Horwedel 1990); (2) direct perturbation; and (3) a graphics package built into RESRAD that shows parameter sensitivities while RESRAD is operational. 


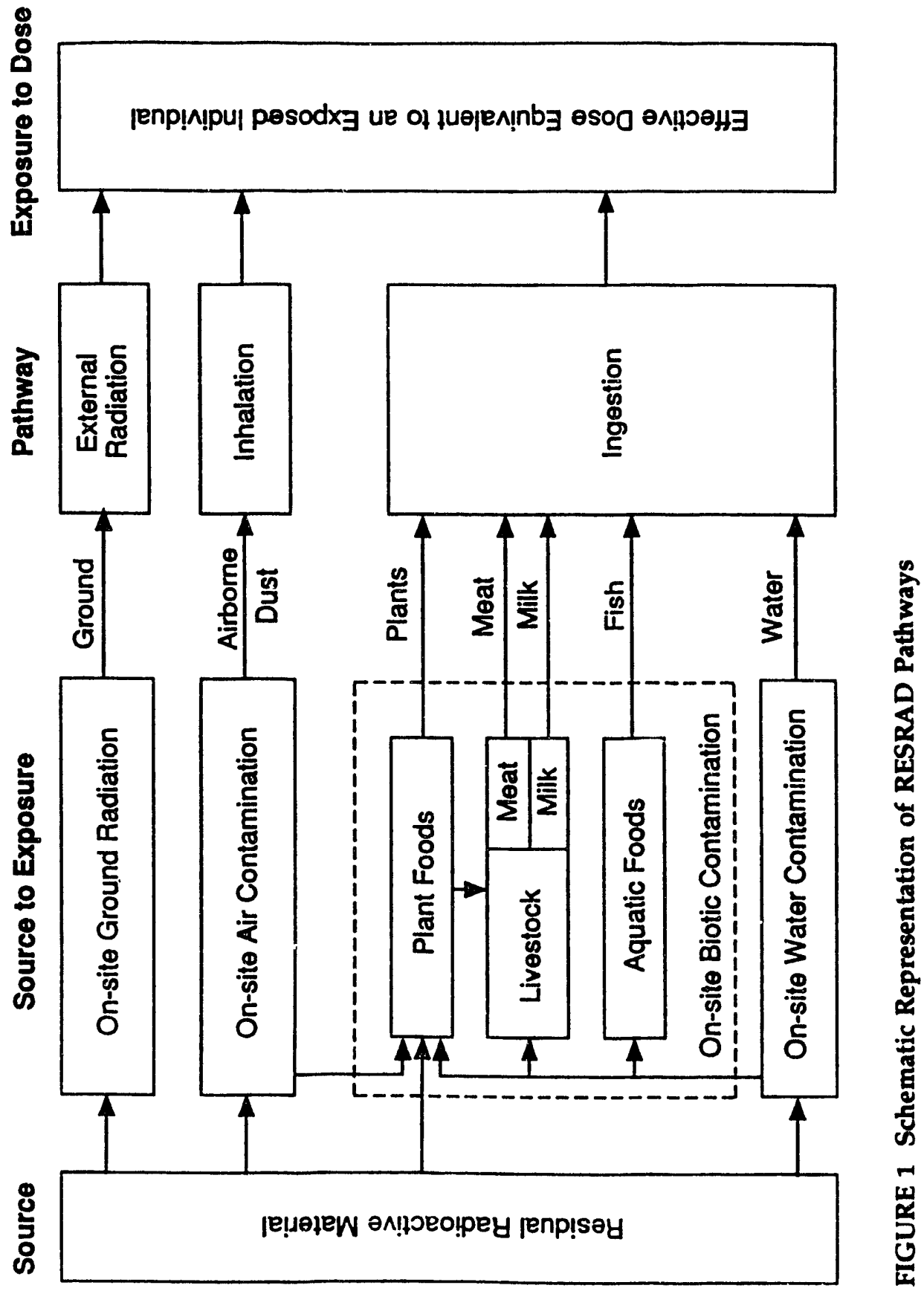


Section 2 of this report describes the sensitivity analysis performed via the GRESS enhancement. In Section 3, use of direct perturbation to verify the accuracy of the GRESS enhancement results is described. Section 4 describes use of the graphics package of RESRAD 4.0 for sensitivity analysis. Results and conclusions are discussed in Section 5. 


\section{SENSITIVITY ANALYSIS VIA THE GRESS SOFTWARE PACKAGE}

In this section, the sensitivity analysis performed via the GRESS enhancement of the RESRAD code is described. Gradients and sensitivities of the calculated results (responses) with respect to specified parameters are presented.

The GRESS package is a collection of programs that allows the automatic calculation of the sensitivity of the results of a given Fortran program with respect to specified parameters, typically input parameters. The capacity of GRESS to perform a sensitivity analysis is based on the design characteristics of a computer program. Because the calculations in a computer program are sequential, that is, a variable is calculated first for its value and is then used in subsequent calculations, it is always possible to find the derivative of a variable on the left-hand side of an equation with respect to the variables on the right-hand side. With propagation of the calculations and storage of derivative information when each mathematical statement is executed, the sensitivity of the results with respect to specified parameters can finally be obtained. The procedures for using GRESS to perform a sensitivity analysis are described in detail in the GRESS manual (Horwedel 1990).

The GRESS package consists of a precompiler program (EXAP) that enhances a Fortran program; a library that contains numerous subroutines that enable the user to direct the sensitivity analysis process and that enable GRESS to perform the gradient calculations of different mathematical expressions; a program (BREDUCE) that reduces the size of the gradient matrix; and a program (BSOLVE) that inverses the gradient matrix and calculates the sensitivities. The user must first insert some "call" statements to the GRESS subroutines in his or her source program to specify the results of interest for a sensitivity analysis and to direct the analytical process. This source program is then input into the GRESS precompiler for enhancement. During the enhancement process, the precompiler reads each statement in the source code and, if necessary, replaces it with a mathematically equivalent, but GRESS-specific, statement that is usually a call statement to the GRESS library subroutines. Following the enhancement, the enhanced code is compiled, linked with the GRESS library, and then executed. After execution, the normal calculational results of the source code are generated, together with a gradient data file containing sensitivity information. The matrix component of this gradient data file can be reduced dimensionally and solved via execution of the BSOLVE program, which then triggers the sensitivity results.

The primary results from the use of GRESS are numbers that represent the gradients of calculated quantities (responses [R]) with respect to specified input quantities (parameters [P]). The gradients are $d R / d P$, which are perhaps more usefully specified as sensitivities; that is, $(d R / R) /(d P / P)$, which is equivalent to $d(\ln R) / d(\ln P)$. If a response $R$ varies with a parameter $P$, such that $R=$ Const $\cdot P^{s}$, then the sensitivity is $s$. A high sensitivity indicates a strong dependence on the parameter; a low sensitivity indicates a weak dependence. The primary functions of GRESS are the propagation and calculation of these gradients with as little user involvement as possible while each statement of the program is executed. The propagation of derivatives over many executed lines of code requires substantial storage capability and eventually the inversion of a large matrix. For RESRAD, the matrix size is about 58,000 $\times 58,000$. 


\subsection{MODIFICATION OF RESRAD FOR GRESS PRECOMPILATION}

The GRESS package was developed for implementation on a VAX computer. Consequently, the RESRAD code must be converted so that it can be run on a VAX. Fortran statements that have different expressions in a VAX computer, such as intrinsic subroutine names, must be converted so that an accurate date and time are obtained for the output. The precision specification for a real number must also be converted to avoid any compilation errors.

When the same input file, that is, SENSE.DAT, was used, the same numerical output was obtained for the VAX version of RESRAD as for the personal computer version. However, the variable values calculated during the iteration procedures by using Brent's method (Press et al. 1989) were sometimes slightly different, but within the expected error range. This slight variation is due to the difference in precision between the two computer systems.

The RESRAD code (VAX version) must be further modified to perform a sensitivity analysis. Calls to several GRESS subroutines must be inserted into RESRAD to specify the results of interest for sensitivity analysis, to direct the GRESS calculational procedure whereby derivatives of mathematical statements are obtained, and to store this information throughout the RESRAD program.

Once the statement of code is executed, GRESS calculates and? stores gradients for each mathematical statement. Because of this accumulational characteristic, any trial-and-error calculational procedure may cause error in the sensitivity analysis if it is enhanced and its gradient information passed on throughout the program. Version 3.121A of RESRAD includes three calculational laps in each execution. The first lap calculates the dose/source ratio and total effective dose for each principal radionuclide at user-specified years. Lap 2 uses iterative calculations to determine the years when the minimum soil cleanup criteria for each radionuclide can be derived. Iterative calculations are also used in Lap 3 to determine the year when the maximum dose/source ratio for all radionuclides will occur. Because the iterative calculations (based on Brent's method) are actually trial-and-error procedures to find the values that best fit the data, the mathematical statements involved are purely numerical analyses without any physical basis. When RESRAD was enhanced by GRESS, only the results from Lap 1 were specified as results of interest because of the trial-and-error procedures in Laps 2 and 3; therefore, only the sensitivities of the results from Lap 1 were output and printed. The results of interest that were chosen were TDOSE(IY) - the total dose (TDOSE [mrem/yr]) at a specified year (T[IY]) and DSR(IY,IR,I) ([mrem/yr]/[pCi/g]) - the dose/source ratio for pathway I and radionuclide IR at a specified year (T[IY]).

The implementation of analytical differentiation in the GRESS methodology may also cause error if the sensitivity analysis involves the derivation of gradients for a discontinuous response function. In this case, the gradients are given either a zero or a wrong value. To overcome this problem, the factor that might have resulted in discontinuity in the calculation was excluded from the sensitivity analysis. Nonzero erosion rates of the cover material and the contaminated zone can cause a sudden disappearance of these two soil layers from one specified year to the next and result in an abrupt change in the gradient values. Under certain circumstances in the RESRAD model, a nonzero value for the water table drop rate will cause a sudden increase in the breakthrough time of ground water contamination from zero to some nonzero value. Therefore, the erosion rates of the cover material and contaminated zone and the water table drop rate were assigned a value of zero. The input data file SENSE.DAT, which was used for sensitivity analysis, is provided in Appendix $C$. 


\subsection{GRESS ENHANCEMENT SENSITIVITY ANALYSIS RESULTS}

The calculated sensitivities of the parameters are printed out in the program BSOLVE, which is part of GRESS. The BSOLVE routine was modified slightly so that it could sort the calculated sensitivities, if desired, and print a more detailed description of the response variables. Typical results for a specific variable, TDOSE(8), are provided in Appendix D.

Tables 1 through 8 give the derivatives and sensitivities of selected variables. Before the breakthrough time of the groundwater contamination (401.3 years in this case), the total dose, for which water-independent pathways are the primary contributors, decreases as time increases, as can be seen in the summary report presented in Appendix E. After the breakthrough timie, the dose contributions from water-dependent pathways play a dominant role, resulting in a maximum total dose at 1406.3 years. Because of the changing dominancy in contributing pathways, parameter sensitivities to the total dose can vary from time to time. Tables 1 and 2 provide parameter sensitivities before radionuclides reach the groundwater table (401.3 years). Values obtained by the direct perturbation method are also provided for comparison with GRESS enhancement results. Parameters such as COVER0 -- thickness of cover material $(0.5 \mathrm{~m})$, THICKO -- thickness of contaminated zone $(1 \mathrm{~m})$, DROOT -- depth of plant root $(0.9 \mathrm{~m})$,

TABLE 1 Sensitivity Analysis of Total Dose at 100 Years (TDOSE[6])

\begin{tabular}{|c|c|c|c|c|}
\hline \multirow[b]{3}{*}{$\begin{array}{c}\text { Input } \\
\text { Parameters }\end{array}$} & \multicolumn{4}{|c|}{$\operatorname{TDOSE}(6)=3.446 \times 10^{-2} \mathrm{mrem} / \mathrm{yr}$} \\
\hline & \multicolumn{2}{|c|}{ Derivatives } & \multicolumn{2}{|c|}{ Sensitivities } \\
\hline & $\begin{array}{c}\text { GRESS } \\
\text { Enhancement }\end{array}$ & $\begin{array}{c}\text { Direct } \\
\text { Perturbation }\end{array}$ & $\begin{array}{c}\text { GRESS } \\
\text { Enharicement }\end{array}$ & $\begin{array}{c}\text { Direct } \\
\text { Perturbation }\end{array}$ \\
\hline COVEROb & $-8.695 \times 10^{-2}$ & $-8.695 \times 10^{-2}$ & $-1.262 \times 10^{0}$ & $-1.261 \times 10^{0}$ \\
\hline DROOT & $4.776 \times 10^{-2}$ & $4.772 \times 10^{-2}$ & $1.247 \times 10^{0}$ & $1.246 \times 10^{0}$ \\
\hline DIET(1) & $1.734 \times 10^{-2}$ & $1.734 \times 10^{-2}$ & $8.049 \times 10^{-1}$ & $8.049 \times 10^{-1}$ \\
\hline EVAPTR & $4.277 \times 10^{-2}$ & $4.278 \times 10^{-2}$ & $7.446 \times 10^{-1}$ & $7.449 \times 10^{-1}$ \\
\hline$S(39)^{c}$ & $1.763 \times 10^{-2}$ & $1 . \% 64 \times 10^{-2}$ & $5.116 \times 10^{-1}$ & $5.117 \times 10^{-1}$ \\
\hline THICKOC & $1.711 \times 10^{-2}$ & $1.710 \times 10^{-2}$ & $4.965 \times 10^{-1}$ & $4.963 \times 10^{-1}$ \\
\hline DENSCZ $^{c}$ & $1.064 \times 10^{-2}$ & $1.064 \times 10^{-2}$ & $4.941 \times 10^{-1}$ & $4.938 \times 10^{-1}$ \\
\hline$S(42)^{c}$ & $1.683 \times 10^{-2}$ & $1.683 \times 10^{-2}$ & $4.884 \times 10^{-1}$ & $4.885 \times 10^{-1}$ \\
\hline PRECIP & $-1.369 \times 10^{-2}$ & $-1.370 \times 10^{-2}$ & $-3.971 \times 10^{-1}$ & $-3.969 \times 10^{-1}$ \\
\hline DCACTC $(39)^{\mathrm{C}}$ & $1.735 \times 10^{-4}$ & $1.735 \times 10^{-4}$ & $2.517 \times 10^{-1}$ & $2.516 \times 10^{-1}$ \\
\hline $\operatorname{DCACTC}(42)^{\mathrm{C}}$ & $1.670 \times 10^{-4}$ & $1.669 \times 10^{-4}$ & $2.423 \times 10^{-1}$ & $2.421 \times 10^{-1}$ \\
\hline $\mathrm{AREA}^{\mathrm{c}}$ & $4.222 \times 10^{-7}$ & $4.224 \times 10^{-7}$ & $1.225 \times 10^{-1}$ & $1.226 \times 10^{-1}$ \\
\hline $\operatorname{DIET}(4)$ & $5.872 \times 10^{-5}$ & $5.872 \times 10^{-5}$ & $1.073 \times 10^{-1}$ & $1.073 \times 10^{-1}$ \\
\hline LFI5 & $5.440 \times 10^{-5}$ & $5.440 \times 10^{-5}$ & $1.073 \times 10^{-1}$ & $1.073 \times 10^{-1}$ \\
\hline
\end{tabular}

a Only pararneters with an absolute sensitivity greater than 0.1 are listed.

${ }^{b}$ Parameter associated with cover material.

'Parameter associated with contaminated zone. 
TABLE 2 Sensitivity Analysis of Total Dose at 300 Years (TDOSE[7]) $^{2}$

\begin{tabular}{|c|c|c|c|c|}
\hline \multirow[b]{3}{*}{$\begin{array}{c}\text { Input } \\
\text { Parameters }\end{array}$} & \multicolumn{4}{|c|}{$\operatorname{TDOSE}(7)=1.390 \times 10^{-2} \mathrm{mrem} / \mathrm{yr}$} \\
\hline & \multicolumn{2}{|c|}{ Derivatives } & \multicolumn{2}{|c|}{ Sensitivities } \\
\hline & $\begin{array}{c}\text { GRESS } \\
\text { Enhancement }\end{array}$ & $\begin{array}{c}\text { Direct } \\
\text { Perturbation }\end{array}$ & $\begin{array}{c}\text { GRESS } \\
\text { Enhancement }\end{array}$ & $\begin{array}{c}\text { Direct } \\
\text { Perturbation }\end{array}$ \\
\hline EVAPTR & $4.953 \times 10^{-2}$ & $4.958 \times 10^{-2}$ & $2.138 \times 10^{0}$ & $2.141 \times 10^{0}$ \\
\hline THICKO & $1.982 \times 10^{-2}$ & $1.981 \times 10^{-2}$ & $1.426 \times 10^{0}$ & $1.426 \times 10^{0}$ \\
\hline DENSCZC & $1.233 \times 10^{-2}$ & $1.233 \times 10^{-2}$ & $1.420 \times 10^{0}$ & $1.420 \times 10^{0}$ \\
\hline COVERO ${ }^{\mathrm{b}}$ & $-3.506 \times 10^{-2}$ & $-3.506 \times 10^{-2}$ & $-1.262 \times 10^{0}$ & $-1.261 \times 10^{0}$ \\
\hline DROOT & $1.926 \times 10^{-2}$ & $1.924 \times 10^{-2}$ & $1.247 \times 10^{0}$ & $1.246 \times 10^{0}$ \\
\hline PRECIP & $-1.585 \times 10^{-2}$ & $-1.584 \times 10^{-2}$ & $-1.140 \times 10^{0}$ & $-1.140 \times 10^{0}$ \\
\hline $\operatorname{DIET}(1)$ & $7.051 \times 10^{-5}$ & $7.051 \times 10^{-5}$ & $8.118 \times 10^{-1}$ & $8.118 \times 10^{-1}$ \\
\hline DCACTC $(39)^{c}$ & $2.017 \times 10^{-4}$ & $2.016 \times 10^{-4}$ & $7.256 \times 10^{-1}$ & $7.254 \times 10^{-1}$ \\
\hline DCACTC $(42)^{c}$ & $1.850 \times 10^{-4}$ & $1.850 \times 10^{-4}$ & $6.657 \times 10^{-1}$ & $6.657 \times 10^{-1}$ \\
\hline$S(39)^{c}$ & $7.677 \times 10^{-3}$ & $7.678 \times 10^{-3}$ & $5.524 \times 10^{-1}$ & $5.525 \times 10^{-1}$ \\
\hline$S(42)^{c}$ & $6.220 \times 10^{-3}$ & $6.221 \times 10^{-3}$ & $4.476 \times 10^{-1}$ & $4.477 \times 10^{-1}$ \\
\hline RI & $-1.981 \times 10^{-2}$ & $-1.982 \times 10^{-2}$ & $-2.851 \times 10^{-1}$ & $-2.852 \times 10^{-1}$ \\
\hline RUNOFF & $1.981 \times 10^{-2}$ & $1.982 \times 10^{-2}$ & $2.851 \times 10^{-1}$ & $2.852 \times 10^{-1}$ \\
\hline $\mathrm{AREA}^{\mathrm{c}}$ & $1.597 \times 10^{-7}$ & $1.596 \times 10^{-7}$ & $1.149 \times 10^{-1}$ & $1.149 \times 10^{-1}$ \\
\hline DIET(4) & $2.228 \times 10^{-5}$ & $2.228 \times 10^{-5}$ & $1.010 \times 10^{-1}$ & $1.010 \times 10^{-1}$ \\
\hline LFI5 & $2.064 \times 10^{-5}$ & $2.064 \times 10^{-5}$ & $1.010 \times 10^{-1}$ & $1.010 \times 10^{-1}$ \\
\hline
\end{tabular}

a Only parameters with an absolute sensitivity greater than 0.1 are listed.

${ }^{\text {b} P a r a m e t e r ~ a s s o c i a t e d ~ w i t h ~ c o v e r ~ m a t e r i a l . ~}$

cParameter associated with contaminated zone.

DENSCZ - density of contaminated zone $\left(1.6 \mathrm{~g} / \mathrm{cm}^{3}\right)$, and DCACTC -- distribution coefficient in contaminated zone $\left(50 \mathrm{~cm}^{3} / \mathrm{g}\right.$ for uranium-234 and uranium-238) have more influence than parameters such as DWIBWT -- well intake depth below groundwater table $(10 \mathrm{~m})$, EPSZ -effective porosity of saturated zone (0.2), DENSAQ -- density of saturated zone $\left(1.6 \mathrm{~g} / \mathrm{cm}^{3}\right)$, and DCACTS -- distribution coefficient in saturated zone $\left(50 \mathrm{~cm}^{3} / \mathrm{g}\right.$ for uranium-234 and uranium-238), because of larger sensitivities. Parameter definitions can be found in Appendixes $\mathrm{A}$ and $\mathrm{B}$. As expected, parameters describing the characteristics of the unsaturated and saturated zones play an important role after radionuclides break through the unsaturated zone and reach the groundwater table (saturated zone). In Table 3, the sensitivities of the parameters of the saturated zone are greater than those of the unsaturated zone before the occurrence of the maximum total dose (1406.3 years); in Table 4, the sensitivities of the parameters of the unsaturated zone greatly increase and are comparable with those of the saturated zone after the occurrence of the maximum total dose. A closer look at the magnitudes of these parameters (for both unsaturated and saturated zones in Tables 3 and 4) reveals opposite signs for the same parameters. The rise time (i.e., the time that the maximum radionuclide concentration occurs in groundwater) can be viewed as the divider between the accumulation and depletion processes of the radionuclides in the groundwater pathway. Before 
TABLE 3 Sensitivity Analysis of Total Dose at 1000 Years (TDOSE[8]) ${ }^{\mathbf{a}}$

\begin{tabular}{|c|c|c|c|c|}
\hline \multirow[b]{3}{*}{$\begin{array}{c}\text { Input } \\
\text { Parameters }\end{array}$} & \multicolumn{4}{|c|}{$\operatorname{TDOSE}(8)=9.650 \times 10^{-1} \mathrm{mrem} / \mathrm{yr}$} \\
\hline & \multicolumn{2}{|c|}{ Derivatives } & \multicolumn{2}{|c|}{ Sensitivities } \\
\hline & $\begin{array}{c}\text { GRESS } \\
\text { Enhancement }\end{array}$ & $\begin{array}{c}\text { Direct } \\
\text { Perturbation }\end{array}$ & $\begin{array}{c}\text { GRESS } \\
\text { Enhancement }\end{array}$ & $\begin{array}{c}\text { Direct } \\
\text { Perturbation }\end{array}$ \\
\hline DWIBWT $^{d}$ & $-9.614 \times 10^{-2}$ & $-9.605 \times 10^{-2}$ & $-9.963 \times 10^{-1}$ & $-9.953 \times 10^{-1}$ \\
\hline EPSZ $^{\mathrm{d}}$ & $-4.807 \times 10^{0}$ & $-4.802 \times 10^{0}$ & $-9.963 \times 10^{-1}$ & $-9.953 \times 10^{-1}$ \\
\hline DENSAQ ${ }^{d}$ & $-5.979 \times 10^{-1}$ & $-5.974 \times 10^{-1}$ & $-9.913 \times 10^{-1}$ & $-9.904 \times 10^{-1}$ \\
\hline TPSZ $^{\mathrm{d}}$ & $2.392 \times 10^{0}$ & $2.391 \times 10^{0}$ & $9.913 \times 10^{-1}$ & $9.913 \times 10^{-1}$ \\
\hline DENSCZ & $5.074 \times 10^{-1}$ & $5.067 \times 10^{-1}$ & $8.413 \times 10^{-1}$ & $8.401 \times 10^{-1}$ \\
\hline THICKO ${ }^{b}$ & $8.113 \times 10^{-1}$ & $8.101 \times 10^{-1}$ & $8.407 \times 10^{-1}$ & $8.395 \times 10^{-1}$ \\
\hline FDW & $7.952 \times 10^{-1}$ & $7.952 \times 10^{-1}$ & $8.240 \times 10^{-1}$ & $8.241 \times 10^{-1}$ \\
\hline DWI & $1.939 \times 10^{-3}$ & $1.939 \times 10^{-3}$ & $8.240 \times 10^{-1}$ & $8.210 \times 10^{-1}$ \\
\hline FGWDW & $7.872 \times 10^{-1}$ & $7.873 \times 10^{-1}$ & $8.158 \times 10^{-1}$ & $8.158 \times 10^{-1}$ \\
\hline$S(39)^{b}$ & $4.961 \times 10^{-1}$ & $4.961 \times 10^{-1}$ & $5.141 \times 10^{-1}$ & $5.141 \times 10^{-1}$ \\
\hline DCACTS(39) d & $-9.672 \times 10^{-3}$ & $-9.663 \times 10^{-3}$ & $-5.011 \times 10^{-1}$ & $-5.007 \times 10^{-1}$ \\
\hline$S(42)^{b}$ & $4.689 \times 10^{-1}$ & $4.690 \times 10^{-1}$ & $4.859 \times 10^{-1}$ & $4.859 \times 10^{-1}$ \\
\hline $\operatorname{DCACTS}(42)^{d}$ & $-9.300 \times 10^{-3}$ & $-9.292 \times 10^{-3}$ & $-4.819 \times 10^{-1}$ & $-4.815 \times 10^{-1}$ \\
\hline EVAPTR & $-6.801 \times 10^{-1}$ & $-6.845 \times 10^{-1}$ & $-4.229 \times 10^{-1}$ & $-4.256 \times 10^{-}$ \\
\hline PRECIP & $2.176 \times 10^{-1}$ & $2.165 \times 10^{-1}$ & $2.255 \times 10^{-1}$ & $2.244 \times 10^{-1}$ \\
\hline RI & $9.815 \times 10^{-1}$ & $9.751 \times 10^{-1}$ & $2.034 \times 10^{-1}$ & $2.021 \times 10^{-1}$ \\
\hline FGWIR & $1.405 \times 10^{-1}$ & $1.404 \times 10^{-1}$ & $1.456 \times 10^{-1}$ & $1.455 \times 10^{-1}$ \\
\hline$H(1)^{c}$ & $-3.050 \times 10^{-2}$ & $-3.055 \times 10^{-2}$ & $-1.264 \times 10^{-1}$ & $-1.266 \times 10^{-1}$ \\
\hline $\operatorname{EPUZ}(1)^{c}$ & $-6.101 \times 10^{-1}$ & $-6.109 \times 10^{-1}$ & $-1.264 \times 10^{-1}$ & $-1.266 \times 10^{-1}$ \\
\hline TPUZ(1) & $3.041 \times 10^{-1}$ & $3.035 \times 10^{-1}$ & $1.261 \times 10^{-1}$ & $1.258 \times 10^{-1}$ \\
\hline DENSUZ(1) ${ }^{c}$ & $-7.603 \times 10^{-2}$ & $-7.611 \times 10^{-2}$ & $-1.261 \times 10^{-1}$ & $-1.262 \times 10^{-1}$ \\
\hline
\end{tabular}

anly parameters with an absolute sensitivity greater than 0.1 are listed.

bParameter associated with contaminated zone.

'Parameter associated with unsaturated zone.

${ }^{\mathrm{d}}$ Parameter associated with saturated zone.

the rise time (after the breakthrough time), the amount of radionuclides leaching through the saturated zone to a well located at the downgradient edge of the contaminated site is greater than that in water drawn from the well. Therefore, the concentration of radionuclides in the groundwater at the point of use will increase with time. After the rise time, the amount of the inflow and outflow of radionuclides reverses, causing the groundwater concentration in the well to decrease. Four parameters strongly influence the total dose regardless of whether they appear before or after the breakthrough time: EVAPTR -- the evapotranspiration coefficient (0.6), PRECIP -- the annual precipitation rate $(1 \mathrm{~m} / \mathrm{yr}), \mathrm{RI}$-- the annual irrigation rate $(0.2 \mathrm{~m} / \mathrm{yr})$, and RUNOFF - the runoff coefficient (0.2). The water infiltration rate, which is calculated on the basis of these four parameters, affects the amount of radionuclides transferred from the contaminated zone to the groundwater in the leaching process. These important parameters are also included in Tables 1 to 4 . 
TABLE 4 Sensitivity Analysis of Total Dose at 3000 Years (TDOSE[9]) ${ }^{a}$

\begin{tabular}{|c|c|c|c|c|}
\hline \multirow[b]{3}{*}{$\begin{array}{c}\text { Input } \\
\text { Parameters }\end{array}$} & \multicolumn{4}{|c|}{$\operatorname{TDOSE}(9)=7.425 \times 10^{-2} \mathrm{mrem} / \mathrm{yr}$} \\
\hline & \multicolumn{2}{|c|}{ Derivatives } & \multicolumn{2}{|c|}{ Sensitivities } \\
\hline & $\begin{array}{c}\text { GRESS } \\
\text { Enhancement }\end{array}$ & $\begin{array}{c}\text { Direct } \\
\text { Perturbation }\end{array}$ & $\begin{array}{c}\text { GRESS } \\
\text { Enhancement }\end{array}$ & $\begin{array}{c}\text { Direct } \\
\text { Perturbation }\end{array}$ \\
\hline EVAPTR & $1.353 \times 10^{0}$ & $1.358 \times 10^{0}$ & $1.093 \times 10^{1}$ & $1.098 \times 10^{1}$ \\
\hline PRECIP & $-4.329 \times 10^{-1}$ & $-4.341 \times 10^{-1}$ & $-5.831 \times 10^{0}$ & $-5.847 \times 10^{0}$ \\
\hline DWIBWT $^{d}$ & $2.862 \times 10^{-2}$ & $2.868 \times 10^{-2}$ & $3.854 \times 10^{0}$ & $3.862 \times 10^{0}$ \\
\hline EPSZ $^{d}$ & $1.431 \times 10^{0}$ & $1.434 \times 10^{0}$ & $3.854 \times 10^{0}$ & $3.862 \times 10^{0}$ \\
\hline DENSAQ & $1.784 \times 10^{-1}$ & $1.788 \times 10^{-1}$ & $3.844 \times 10^{0}$ & $3.852 \times 10^{0}$ \\
\hline TPSZ $^{\mathrm{d}}$ & $-7.136 \times 10^{-1}$ & $-7.114 \times 10^{-1}$ & $-3.844 \times 10^{0}$ & $-3.833 \times 10^{0}$ \\
\hline $\operatorname{DCACTS}(22)^{d}$ & $2.823 \times 10^{-3}$ & $2.828 \times 10^{-3}$ & $3.802 \times 10^{0}$ & $3.809 \times 10^{0}$ \\
\hline $\operatorname{EPUZ}(1)^{\mathrm{c}}$ & $7.087 \times 10^{-1}$ & $7.096 \times 10^{-1}$ & $1.909 \times 10^{0}$ & $1.911 \times 10^{0}$ \\
\hline $\mathrm{H}(1)^{\mathrm{c}}$ & $3.544 \times 13^{-2}$ & $3.548 \times 10^{-2}$ & $1.909 \times 10^{0}$ & $1.911 \times 10^{0}$ \\
\hline TPUZ(1) ${ }^{c}$ & $-3.538 \times 0^{-1}$ & $-3.530 \times 10^{-1}$ & $-1.906 \times 10^{0}$ & $-1.902 \times 10^{0}$ \\
\hline DENSUZ(1) ${ }^{c}$ & $8.844 \times 10^{-2}$ & $8.853 \times 10^{-2}$ & $1.906 \times 10^{0}$ & $1.908 \times 10^{0}$ \\
\hline $\operatorname{DCACTU}(22,1)^{c}$ & $1.399 \times 10^{-3}$ & $1.401 \times 10^{-3}$ & $1.885 \times 10^{0}$ & $1.887 \times 10^{0}$ \\
\hline THICKO ${ }^{\mathrm{b}}$ & $1.133 \times 10^{-1}$ & $1.136 \times 10^{-1}$ & $1.526 \times 10^{0}$ & $1.530 \times 10^{0}$ \\
\hline DENSCZ $^{\mathrm{b}}$ & $7.074 \times 10^{-2}$ & $7.088 \times 10^{-2}$ & $1.524 \times 10^{0}$ & $1.527 \times 10^{0}$ \\
\hline RUNOFF & $5.412 \times 10^{-1}$ & $5.313 \times 10^{-1}$ & $1.458 \times 10^{0}$ & $1.431 \times 10^{0}$ \\
\hline $\mathrm{RI}$ & $-3.866 \times 10^{-1}$ & $-3.943 \times 10^{-1}$ & $-1.041 \times 10^{0}$ & $-1.062 \times 10^{0}$ \\
\hline$S(39)^{b}$ & $7.386 \times 10^{-2}$ & $7.386 \times 10^{-2}$ & $9.947 \times 10^{-1}$ & $9.947 \times 10^{-1}$ \\
\hline DWI & $9.638 \times 10^{-5}$ & $9.639 \times 10^{-5}$ & $5.322 \times 10^{-1}$ & $5.322 \times 10^{-1}$ \\
\hline FDW & $3.952 \times 10^{-2}$ & $3.953 \times 10^{-2}$ & $5.322 \times 10^{-1}$ & $5.323 \times 10^{-1}$ \\
\hline FGWDW & $3.912 \times 10^{-2}$ & $3.913 \times 10^{-2}$ & $5.269 \times 10^{-1}$ & $5.269 \times 10^{-1}$ \\
\hline DCACTC $(22)^{b}$ & $3.168 \times 10^{-4}$ & $3.184 \times 10^{-4}$ & $4.267 \times 10^{-1}$ & $4.289 \times 10^{-1}$ \\
\hline DIET(1) & $1.918 \times 10^{-4}$ & $1.918 \times 10^{-4}$ & $4.134 \times 10^{-1}$ & $4.133 \times 10^{-1}$ \\
\hline FGWIR & $3.060 \times 10^{-2}$ & $3.060 \times 10^{-2}$ & $4.122 \times 10^{-1}$ & $4.121 \times 10^{-1}$ \\
\hline
\end{tabular}

${ }^{a}$ Only parameters with an absolute sensitivity greater than 0.1 are listed.

bParameter associated with contaminated zone.

${ }^{\mathrm{c}}$ Parameter associated with unsaturated zone.

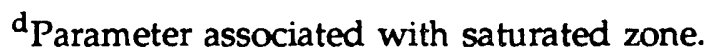

Tables 5 and 6 give the dose/source ratios for uranium-234 in the water-independent plant and ground pathways, respectively. As discussed earlier, prior to the breakthrough time, parameters that are not related to the unsaturated and saturated zones have greater sensitivities because, at this time, the dominant pathways are the water-independent ones. According to the summary report in Appendix $\mathrm{E}$, the water-independent plant pathway contributes the most to the total dose before 401.3 years. Evidence of this is also found in the very similar influential parameters in Tables 1 and 5. Parameters such as COVER0, DROOT, THICK0, DENSCZ, and DCACTC are still listed among the important parameters. Because Table 5 displays the 
TABLE 5 Sensitivity Analysis of Dose/Source Ratio from Uranium-234 in the Water-Independent Plant Pathway at 30 Years (DSR $[5,39,3])^{\mathrm{a}}$

\begin{tabular}{|c|c|c|c|c|}
\hline \multirow[b]{3}{*}{$\begin{array}{c}\text { Input } \\
\text { Parameters }\end{array}$} & \multicolumn{4}{|c|}{$\operatorname{DSR}(5,39,3)=2.167 \times 10^{-2}(\mathrm{mrem} / \mathrm{yr}) /(\mathrm{pCi} / \mathrm{g})$} \\
\hline & \multicolumn{2}{|c|}{ Derivatives } & \multicolumn{2}{|c|}{ Sensitivities } \\
\hline & $\begin{array}{c}\text { GRESS } \\
\text { Enhancement }\end{array}$ & $\begin{array}{c}\text { Direct } \\
\text { Perturbation }\end{array}$ & $\begin{array}{c}\text { GRESS } \\
\text { Enhancement }\end{array}$ & $\begin{array}{c}\text { Direct } \\
\text { Perturbation }\end{array}$ \\
\hline DROOT & $3.010 \times 10^{-2}$ & $3.007 \times 10^{-2}$ & $1.250 \times 10^{0}$ & $1.249 \times 10^{0}$ \\
\hline COVERO ${ }^{b}$ & $-5.418 \times 10^{-2}$ & $-5.418 \times 10^{-2}$ & $-1.250 \times 10^{0}$ & $-1.250 \times 10^{0}$ \\
\hline$S(39)^{c}$ & $2.167 \times 10^{-2}$ & $2.167 \times 10^{-2}$ & $1.000 \times 10^{0}$ & $1.000 \times 10^{0}$ \\
\hline DIET(1) & $1.246 \times 10^{-4}$ & $1.246 \times 10^{-4}$ & $9.195 \times 10^{-1}$ & $9.195 \times 10^{-1}$ \\
\hline EVAPTR & $8.087 \times 10^{-3}$ & $8.087 \times 10^{-3}$ & $2.239 \times 10^{-1}$ & $2.239 \times 10^{-1}$ \\
\hline THICKO & $3.236 \times 10^{-3}$ & $3.232 \times 10^{-3}$ & $1.493 \times 10^{-1}$ & $1.491 \times 10^{-1}$ \\
\hline DENSCZ & $2.015 \times 10^{-3}$ & $2.012 \times 10^{-3}$ & $1.487 \times 10^{-1}$ & $1.485 \times 10^{-1}$ \\
\hline DCACTC $(39)^{c}$ & $6.446 \times 10^{-5}$ & $6.437 \times 10^{-5}$ & $1.487 \times 10^{-1}$ & $1.485 \times 10^{-1}$ \\
\hline PRECIP & $-2.588 \times 10^{-3}$ & $-2.589 \times 10^{-3}$ & $-1.194 \times 10^{-1}$ & $-1.195 \times 10^{-1}$ \\
\hline DIET(2) & $1.246 \times 10^{-3}$ & $1.247 \times 10^{-3}$ & $8.046 \times 10^{-2}$ & $8.053 \times 10^{-2}$ \\
\hline RI & $-3.235 \times 10^{-3}$ & $-3.241 \times 10^{-3}$ & $-2.985 \times 10^{-2}$ & $-2.991 \times 10^{-2}$ \\
\hline RUNOFF & $3.235 \times 10^{-3}$ & $3.232 \times 10^{-3}$ & $2.985 \times 10^{-2}$ & $2.982 \times 10^{-2}$ \\
\hline
\end{tabular}

anly parameters with an absolute sensitivity greater than 0.01 are listed.

barameter associated with cover material.

'Parameter associated with contaminated zone.

dose/source ratio for uranium-234, only the distribution coefficient (DCACTC[39] -- the distribution coefficient of uranium-234 in the contaminated zone $\left[50 \mathrm{~cm}^{3} / \mathrm{g}\right]$ ) for this radionuclide rather than that for both DCACTC(39) and DCACTC(42), which is the distribution coefficient of uranium-238 in the contaminated zone $\left(50 \mathrm{~cm}^{3} / \mathrm{g}\right)$, is observed. In Table 6 , DENSCV -- density of cover material $\left(1.6 \mathrm{~g} / \mathrm{cm}^{3}\right)$-- becomes important because the shielding effect of the cover material reduces the external gamma radiation from the radioactive residues. The appearance of parameter DCACTC $(28)$, the distribution coefficient of radium-226 in the contaminated zone $\left(70 \mathrm{~cm}^{3} / \mathrm{g}\right)$, accounts for the influence of radioactive decay on the intensity of radiation in the ground pathway. Parameter DROOT does not show up because the external radiation has no relation to the depth of the plant root. As in the sensitivity analysis for total dose, no unsaturated or saturated zone parameter is observed in Table 6.

Tables 7 and 8 give the sensitivities of parameters to dose/source ratios for uranium-238 in the groundwater pathway before and after the rise time. The dominant parameters are very similar to those listed for total dose in Tables 3 and 4, which proves the importance of the contribution from the groundwater pathway for variables TDOSE(8) and TDOSE(9). The appearance of parameter DCACTS(22), the water/soil distribution coefficient of lead-210 $\left(100 \mathrm{~cm}^{3} / \mathrm{g}\right)$, in the saturated zone in Table 8 is due to decay of uranium-238 to lead-210. The sensitivities of parameters involved in the leaching process have opposite signs in Tables 7 and 8 , which is also consistent with the results in Tables 3 and 4. 
TABLE 6 Sensitivity Analysis of Dose/Source Ratio from Uranium-234 in the Ground Pathway at 300 Years (DSR $[7,39,3])^{a}$

\begin{tabular}{|c|c|c|c|c|}
\hline \multirow[b]{3}{*}{$\begin{array}{c}\text { Input } \\
\text { Parameters }\end{array}$} & \multicolumn{4}{|c|}{$\operatorname{DSR}(7,39,3)=3.652 \times 10^{-6}(\mathrm{mrem} / \mathrm{yr}) /(\mathrm{pCi} / \mathrm{g})$} \\
\hline & \multicolumn{2}{|c|}{ Derivatives } & \multicolumn{2}{|c|}{ Sensitivities } \\
\hline & $\begin{array}{c}\text { GRESS } \\
\text { Enhancement }\end{array}$ & $\begin{array}{c}\text { Direct } \\
\text { Perturbation }\end{array}$ & $\begin{array}{c}\text { GRESS } \\
\text { Enhancement }\end{array}$ & $\begin{array}{c}\text { Direct } \\
\text { Perturbation }\end{array}$ \\
\hline DENSCV $V^{b}$ & $-1.634 \times 10^{-5}$ & $-1.630 \times 10^{-5}$ & $-7.159 \times 10^{0}$ & $-7.141 \times 10^{0}$ \\
\hline COVERO ${ }^{\mathrm{b}}$ & $-3.859 \times 10^{-5}$ & $-3.849 \times 10^{-5}$ & $-5.284 \times 10^{0}$ & $-5.270 \times 10^{0}$ \\
\hline EVAPTR & $6.827 \times 10^{-6}$ & $6.832 \times 10^{-6}$ & $1.122 \times 10^{0}$ & $1.123 \times 10^{0}$ \\
\hline FO1 & $6.086 \times 10^{-6}$ & $6.086 \times 10^{-6}$ & $1.000 \times 10^{0}$ & $1.000 \times 10^{0}$ \\
\hline$S(39)^{c}$ & $3.652 \times 10^{-6}$ & $3.652 \times 10^{-6}$ & $1.000 \times 10^{0}$ & $1.000 \times 10^{0}$ \\
\hline FS1 & $3.652 \times 10^{-6}$ & $3.652 \times 10^{-6}$ & $1.000 \times 10^{0}$ & $1.000 \times 10^{0}$ \\
\hline THICKO ${ }^{c}$ & $2.733 \times 10^{-6}$ & $2.731 \times 10^{-6}$ & $7.483 \times 10^{-1}$ & $7.478 \times 10^{-1}$ \\
\hline PRECIP & $-2.185 \times 10^{-6}$ & $-2.184 \times 10^{-6}$ & $-5.983 \times 10^{-1}$ & $-5.981 \times 10^{-1}$ \\
\hline DENSCZC & $1.274 \times 10^{-6}$ & $1.272 \times 10^{-6}$ & $5.582 \times 10^{-1}$ & $5.575 \times 10^{-1}$ \\
\hline DCACTC $(39)^{c}$ & $3.081 \times 10^{-8}$ & $3.078 \times 10^{-8}$ & $4.218 \times 10^{-1}$ & $4.215 \times 10^{-1}$ \\
\hline DCACTC $(28)^{c}$ & $1.686 \times 10^{-8}$ & $1.684 \times 10^{-8}$ & $3.231 \times 10^{-1}$ & $3.229 \times 10^{-1}$ \\
\hline RI & $-2.731 \times 10^{-6}$ & $-2.733 \times 10^{-6}$ & $-1.496 \times 10^{-1}$ & $-1.497 \times 10^{-1}$ \\
\hline RUNOFF & $2.731 \times 10^{-6}$ & $2.732 \times 10^{-6}$ & $1.496 \times 10^{-1}$ & $1.496 \times 10^{-1}$ \\
\hline
\end{tabular}

aOnly parameters with an absolute sensitivity greater than 0.1 are listed.

barameter associated with cover material.

'Parameter associated with contaminated zone. 
TABLE 7 Sensitivity Analysis of Dose/Source Ratio from Uranium-238 in the Drinking Water Pathway at 1000 Years (DSR $[8,42,6])^{\text {a }}$

\begin{tabular}{|c|c|c|c|c|}
\hline \multirow[b]{3}{*}{$\begin{array}{c}\text { Input } \\
\text { Parameters }\end{array}$} & \multicolumn{4}{|c|}{$\operatorname{DSR}(8,42,6)=3.884 \times 10^{-1}(\mathrm{mrem} / \mathrm{yr}) /(\mathrm{pCi} / \mathrm{g})$} \\
\hline & \multicolumn{2}{|c|}{ Derivatives } & \multicolumn{2}{|c|}{ Sensitivities } \\
\hline & $\begin{array}{c}\text { GRESS } \\
\text { Enhancement }\end{array}$ & $\begin{array}{c}\text { Direct } \\
\text { Perturbation }\end{array}$ & $\begin{array}{c}\text { GRESS } \\
\text { Enhancement }\end{array}$ & $\begin{array}{c}\text { Direct } \\
\text { Perturbation }\end{array}$ \\
\hline$S(42)^{b}$ & $3.884 \times 10^{-1}$ & $3.884 \times 10^{-1}$ & $1.000 \times 10^{0}$ & $1.000 \times 10^{0}$ \\
\hline DWIBWT $^{d}$ & $-3.884 \times 10^{-2}$ & $-3.882 \times 10^{-2}$ & $-1.000 \times 10^{0}$ & $-9.990 \times 10^{-1}$ \\
\hline FDW & $3.884 \times 10^{-1}$ & $3.884 \times 10^{-1}$ & $1.000 \times 10^{0}$ & $1.000 \times 10^{0}$ \\
\hline EPSZ $^{d}$ & $-1.942 \times 10^{0}$ & $-1.940 \times 10^{0}$ & $-1.000 \times 10^{0}$ & $-9.990 \times 10^{-1}$ \\
\hline DWI & $9.474 \times 10^{-4}$ & $9.474 \times 10^{-4}$ & $1.000 \times 10^{0}$ & $1.000 \times 10^{0}$ \\
\hline DENSAQ & $-2.416 \times 10^{-1}$ & $-2.413 \times 10^{-1}$ & $-9.950 \times 10^{-1}$ & $-9.941 \times 10^{-1}$ \\
\hline TPSZ $^{\mathrm{d}}$ & $9.662 \times 10^{-1}$ & $9.661 \times 10^{-1}$ & $9.950 \times 10^{-1}$ & $9.949 \times 10^{-1}$ \\
\hline $\operatorname{DCACTS}(42)^{d}$ & $-7.707 \times 10^{-3}$ & $-7.700 \times 10^{-3}$ & $-9.921 \times 10^{-1}$ & $-9.912 \times 10^{-1}$ \\
\hline FGWDW & $3.845 \times 10^{-1}$ & $3.846 \times 10^{-1}$ & $9.900 \times 10^{-1}$ & $9.901 \times 10^{-1}$ \\
\hline DENSC $Z^{b}$ & $2.042 \times 10^{-1}$ & $2.042 \times 10^{-1}$ & $8.413 \times 10^{-1}$ & $8.412 \times 10^{-1}$ \\
\hline THICKO & $3.265 \times 10^{-1}$ & $3.265 \times 10^{-1}$ & $8.407 \times 10^{-1}$ & $8.405 \times 10^{-1}$ \\
\hline EVAPTR & $-2.583 \times 10^{-1}$ & $-2.593 \times 10^{-1}$ & $-3.990 \times 10^{-1}$ & $-4.005 \times 10^{-1}$ \\
\hline PRECIP & $8.266 \times 10^{-2}$ & $8.249 \times 10^{-2}$ & $2.128 \times 10^{-1}$ & $2.124 \times 10^{-1}$ \\
\hline DCACTC $(42)^{b}$ & $-1.229 \times 10^{-3}$ & $-1.229 \times 10^{-3}$ & $-1.582 \times 10^{-1}$ & $-1.582 \times 10^{-1}$ \\
\hline EPUZ $(1)^{c}$ & $-2.074 \times 10^{-1}$ & $-2.076 \times 10^{-1}$ & $-1.068 \times 10^{-1}$ & $-1.069 \times 10^{-1}$ \\
\hline $\mathrm{H}(1)^{\mathrm{c}}$ & $-1.037 \times 10^{-2}$ & $-1.038 \times 10^{-2}$ & $-1.068 \times 10^{-1}$ & $-1.069 \times 10^{-1}$ \\
\hline TPUZ(1) & $1.034 \times 10^{-1}$ & $1.032 \times 10^{-1}$ & $1.065 \times 10^{-1}$ & $1.063 \times 10^{-1}$ \\
\hline DENSUZ $(1)^{c}$ & $-2.584 \times 10^{-2}$ & $-2.587 \times 10^{-2}$ & $-1.065 \times 10^{-1}$ & $-1.066 \times 10^{-1}$ \\
\hline $\operatorname{DCACTU}(42,1)^{\mathrm{C}}$ & $-8.243 \times 10^{-4}$ & $-8.255 \times 10^{-4}$ & $-1.061 \times 10^{-1}$ & $-1.063 \times 10^{-1}$ \\
\hline
\end{tabular}

aOnly parameters with an absolute sensitivity greater than 0.1 are listed.

bParameter associated with contaminated zone.

'Parameter associated with unsaturated zone.

dParameter associated with saturated zone. 
TABLE 8 Sensitivity Analysis of Dose/Source Ratio from Uranium-238 in the Drinking Water Pathway at 3000 Years (DSR $[9,42,6])^{a}$

\begin{tabular}{|c|c|c|c|c|}
\hline \multirow[b]{3}{*}{$\begin{array}{c}\text { Input } \\
\text { Parameters }\end{array}$} & \multicolumn{4}{|c|}{$\operatorname{DSR}(9,42,6)=2.666 \times 10^{-4}(\mathrm{mrem} / \mathrm{yr}) /(\mathrm{pCi} / \mathrm{g})$} \\
\hline & \multicolumn{2}{|c|}{ Derivatives } & \multicolumn{2}{|c|}{ Sensitivities } \\
\hline & $\begin{array}{c}\text { GRESS } \\
\text { Enhancement }\end{array}$ & $\begin{array}{c}\text { Direct } \\
\text { Perturbation }\end{array}$ & $\begin{array}{c}\text { GRESS } \\
\text { Enhancement }\end{array}$ & $\begin{array}{c}\text { Direct } \\
\text { Perturbation }\end{array}$ \\
\hline EVAPTR & $7.719 \times 10^{-3}$ & $7.794 \times 10^{-3}$ & $1.737 \times 10^{1}$ & $1.754 \times 10^{1}$ \\
\hline PRECIP & $-2.470 \times 10^{-3}$ & $-2.458 \times 10^{-3}$ & $-9.266 \times 10^{0}$ & $-9.219 \times 10^{0}$ \\
\hline THICKO $0^{\mathrm{b}}$ & $1.479 \times 10^{-1}$ & $1.483 \times 10^{-1}$ & $5.547 \times 10^{0}$ & $5.564 \times 10^{0}$ \\
\hline DENSCZ $^{\mathrm{b}}$ & $9.213 \times 10^{-4}$ & $9.242 \times 10^{-4}$ & $5.529 \times 10^{0}$ & $5.547 \times 10^{0}$ \\
\hline DCACTC $(42)^{b}$ & $2.279 \times 10^{-5}$ & $2.286 \times 10^{-5}$ & $4.275 \times 10^{0}$ & $4.288 \times 10^{0}$ \\
\hline DWIBWT ${ }^{d}$ & $1.076 \times 10^{-4}$ & $1.079 \times 10^{-4}$ & $4.038 \times 10^{0}$ & $4.047 \times 10^{0}$ \\
\hline EPSZ $^{d}$ & $5.382 \times 10^{-3}$ & $5.394 \times 10^{-3}$ & $4.038 \times 10^{0}$ & $4.047 \times 10^{0}$ \\
\hline DENSAQ $^{d}$ & $6.702 \times 10^{-4}$ & $6.716 \times 10^{-4}$ & $4.022 \times 10^{0}$ & $4.031 \times 10^{0}$ \\
\hline TPSZ $^{\mathrm{d}}$ & $-2.681 \times 10^{-3}$ & $-2.673 \times 10^{-3}$ & $-4.022 \times 10^{0}$ & $-4.010 \times 10^{0}$ \\
\hline RI & $-3.088 \times 10^{-3}$ & $-3.085 \times 10^{-3}$ & $-2.316 \times 10^{0}$ & $-2.315 \times 10^{0}$ \\
\hline RUNOFF & $3.088 \times 10^{-3}$ & $3.092 \times 10^{-3}$ & $2.316 \times 10^{0}$ & $2.319 \times 10^{0}$ \\
\hline $\operatorname{DCACTS}(42)^{d}$ & $1.164 \times 10^{-5}$ & $1.166 \times 10^{-5}$ & $2.183 \times 10^{0}$ & $2.187 \times 10^{0}$ \\
\hline $\operatorname{EPUZ}(1)^{c}$ & $2.665 \times 10^{-3}$ & $2.668 \times 10^{-3}$ & $1.999 \times 10^{0}$ & $2.001 \times 10^{0}$ \\
\hline$H(1)^{c}$ & $1.332 \times 10^{-4}$ & $1.334 \times 10^{-4}$ & $1.999 \times 10^{0}$ & $2.001 \times 10^{0}$ \\
\hline TPUZ(1) & $-1.329 \times 10^{-3}$ & $-1.326 \times 10^{-3}$ & $-1.994 \times 10^{0}$ & $-1.990 \times 10^{0}$ \\
\hline DENSUZ $(1)^{\mathrm{c}}$ & $3.322 \times 10^{-4}$ & $3.325 \times 10^{-4}$ & $1.994 \times 10^{0}$ & $1.996 \times 10^{0}$ \\
\hline $\operatorname{DC} . A C T S(22)^{d}$ & $4.806 \times 10^{-6}$ & $4.816 \times 10^{-6}$ & $1.803 \times 10^{0}$ & $1.806 \times 10^{0}$ \\
\hline $\operatorname{DCACTU}(42,1)^{\mathrm{c}}$ & $5.768 \times 10^{-5}$ & $5.774 \times 10^{-5}$ & $1.082 \times 10^{0}$ & $1.083 \times 10^{0}$ \\
\hline FDW & $2.666 \times 10^{-4}$ & $2.666 \times 10^{-4}$ & $1.000 \times 10^{0}$ & $1.000 \times 10^{0}$ \\
\hline DWI & $6.502 \times 10^{-7}$ & $6.502 \times 10^{-7}$ & $1.000 \times 10^{0}$ & $1.000 \times 10^{0}$ \\
\hline $\mathrm{S}(42)^{\mathrm{b}}$ & $2.666 \times 10^{-4}$ & $2.666 \times 10^{-4}$ & $1.000 \times 10^{0}$ & $1.000 \times 10^{0}$ \\
\hline
\end{tabular}

aOnly parameters with an absolute sensitivity greater than 1 are listed.

bParameter associated with contaminated zone.

cParameter associated with unsaturated zone.

${ }^{\mathrm{d}}$ Parameter associated with saturated zone. 


\section{SENSITIVITY ANALYSIS VIA DIRECT PARAMETER PERTURBATION}

To verify the correctness of sensitivity values calculated via the GRESS enhancement of RESRAD, direct parameter perturbation analyses were also performed by varying the values of input parameters in the data file SENSE.DAT. The results obtained from this method are presented and compared with those from the application of the GRESS enhancement.

\subsection{PERTURBATION OF INPUT PARAMETERS}

The relationship among the resulting quantities (responses) and the input quantities (parameters) in the RESRAD model is quite complicated; it is extremely nonlinear. Consequently, the gradients and sensitivities are not constants but depend on the values of the input parameters. Finite perturbations in the input values may cause deviations in the calculated sensitivities. For parameters that are quite insensitive, a small perturbation may result in only a slight change in the response. Sometimes this slight change is beyond the resolution of the computer calculations, and either a zero sensitivity is calculated or the precision of the sensitivities is greatly reduced. On the basis of these considerations, perturbations for parameters with high or low sensitivities were set differently. A quick look at the GRESS enhancement output provided a preliminary comparison of the magnitudes of the sensitivities. To obtain observable changes in the results, a search procedure was used to find an appropriate perturbation value for each parameter. For highly sensitive parameters, a perturbation value of $0.1 \%$ was used, with an additional $0.1 \%$ in each successive step. Whenever two sequential perturbations gave almost the same sensitivity, the perturbation procedure was terminated and the final value was chosen for the sensitivity. It usually took only a few steps to reach this requirement. For parameters with low sensitivities, a perturbation value of $1 \%$ was used, with an additional $1 \%$ in each successive step; the requirement was met within a perturbation value of $5 \%$.

\subsection{CAlCulational PROCEDURE}

The results of the RESRAD execution were saved in two output files, SUMMARY.OUT and DETAILED.OUT, which are described in detail in the RESRAD user's manual (Gilbert et al. 1989). The data in the output files have values expressed in exponential format down to three digits after the decimal point. This is not precise enough, however, for calculating sensitivities of input parameters with only a $0.1 \%$ to $5 \%$ perturbation. Instead of using the default output files, more precise values (with more significant digits) for the variables of interest (in this case, TDOSE[IY] and DSR[IY,IR,I]) were printed by modifying the FORMAT statements in the RESRAD code. The results were saved in a separate file that was then used to calculate the sensitivities. The flow diagram in Figure 2 depicts the calculational procedure. 


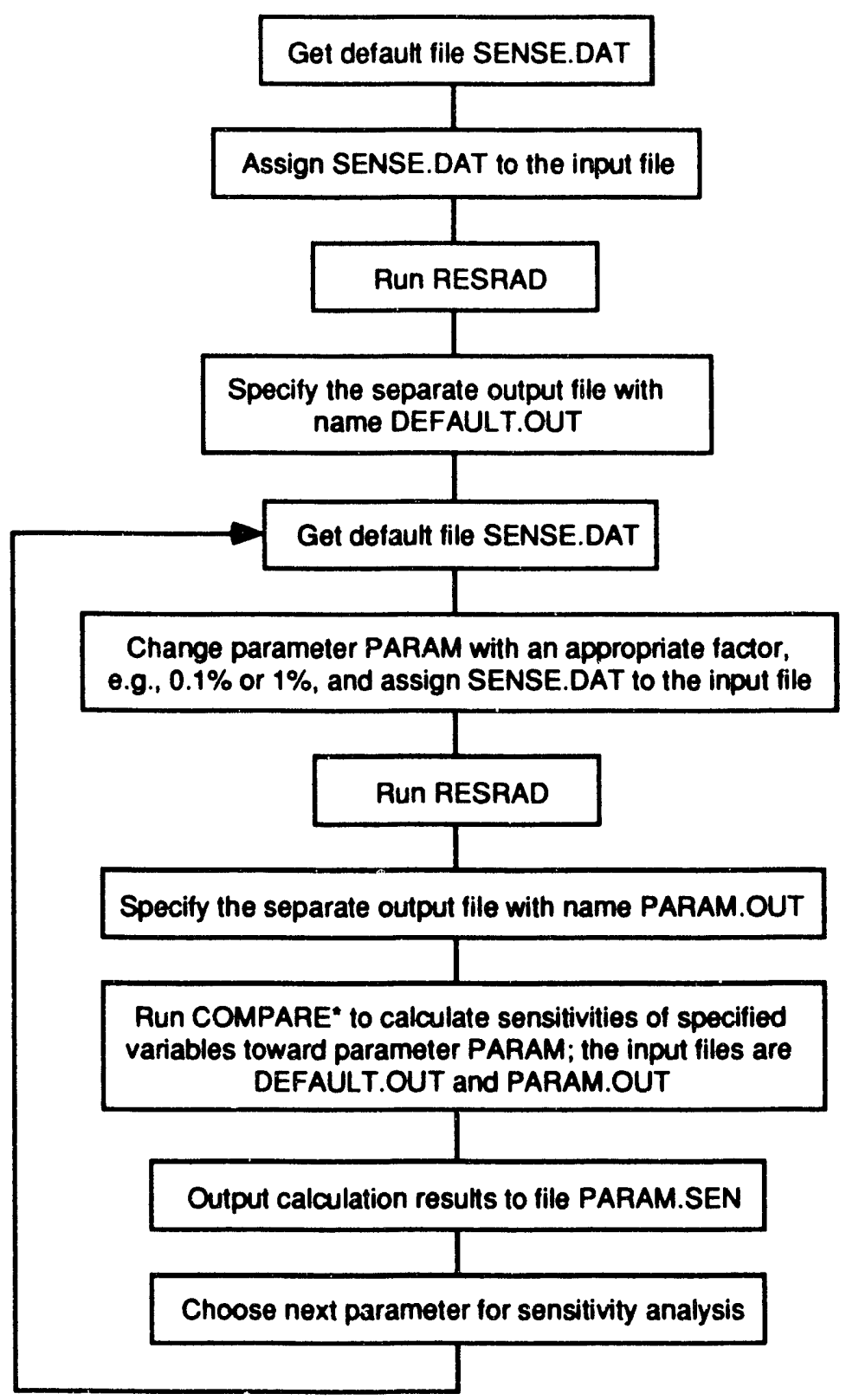

*COMPARE is a program written to calculate the gradients and sensitivities of input parameters.

FIGURE 2 Flow Diagram of Direct Parameter Perturbation Method 


\subsection{COMPARISON OF DIRECT PARAMETER PERTURBATION RESULTS WITH GRESS ENHANCEMENT RESULTS}

The derivatives and sensitivities obtained by the direct parameter perturbation method are given in columns 3 and 5 of Tables 1 through 8; the results from the GRESS enhancement are given in each table for comparison. The results show good agreement. The minor differences in the calculated derivatives and sensitivities between the two methods are due to finite perturbation, which is inevitable in the direct parameter perturbation method. 


\section{SENSITIVITY ANALYSIS VIA RESRAD'S GRAPHICS CAPABILITY}

RESRAD 4.0 can show calculational results in graphic form. Figures 3 and 4 are examples of figures produced by RESRAD 4.0. Figure 3 shows the dose/source ratio for both water-independent and water-dependent pathway subtotals of radionuclide uranium-234; Figure 4 shows the results for individual water-dependent pathways of radionuclide uranium-238. RESRAD 4.0 allows the user to select the parameters for sensitivity analysis, the results of which are then shown graphically in two-dimensional plots.

\subsection{INVOKING SENSITIVITY ANALYSIS FROM THE RESRAD INPUT SCREEN}

RESRAD 4.0 can also perform a sensitivity analysis while operational. For each of the data input screens, the user can select parameters for sensitivity analysis by pressing the F9 key. For example, for the contaminated zone hydrological data input screen (shown in Figure 5), sensitivity analysis for a specific parameter can be invoked by moving the cursor to that parameter and pressing the F9 key. After pressing the F9 key, a screen similar to the one in Figure 6 will appear. Pressing the F10 key when the desired perturbation factor is highlighted will invoke the sensitivity calculation during execution. The input parameters that RESRAD can analyze for sensitivity are listed in Appendix B.

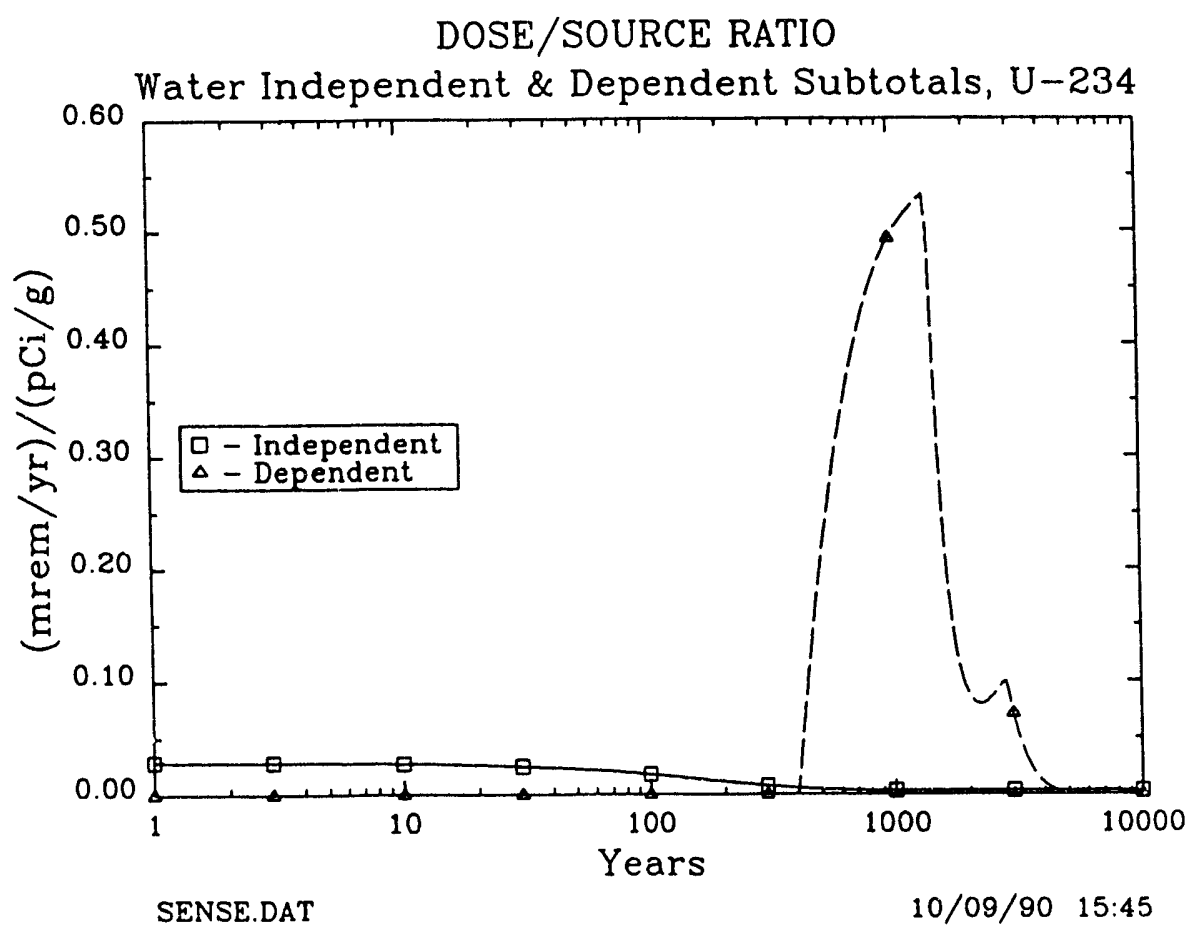

FIGURE 3 Dose/Source Ratio of Water-Independent and WaterDependent Pathway Subtotals of Uranium-234 


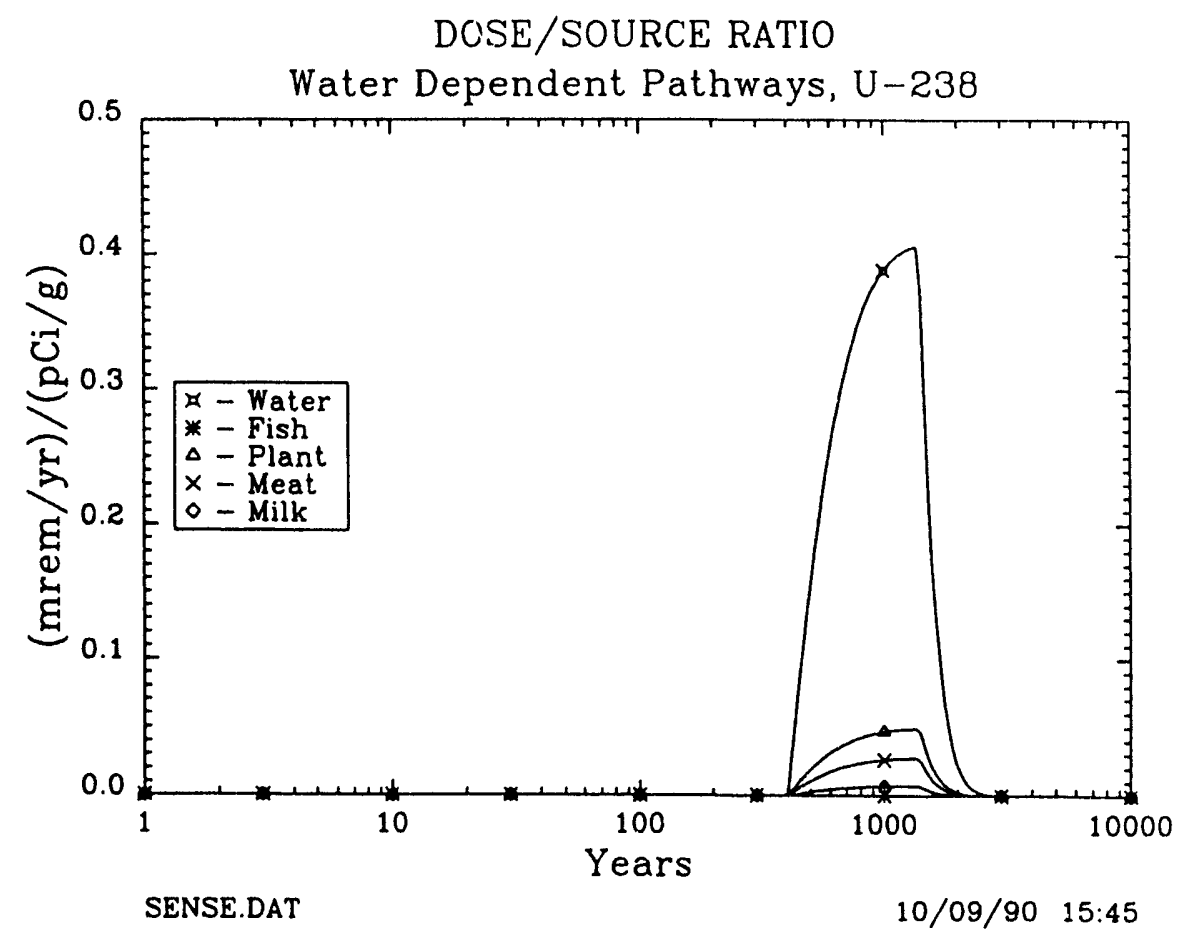

FIGURE 4 Dose/Source Ratio of Water-Dependent Pathways of Uranium-238

RESRAD: Residual Radioactive Material Program $\operatorname{Path}(8): 1,2,3,4,5,6,7,8$

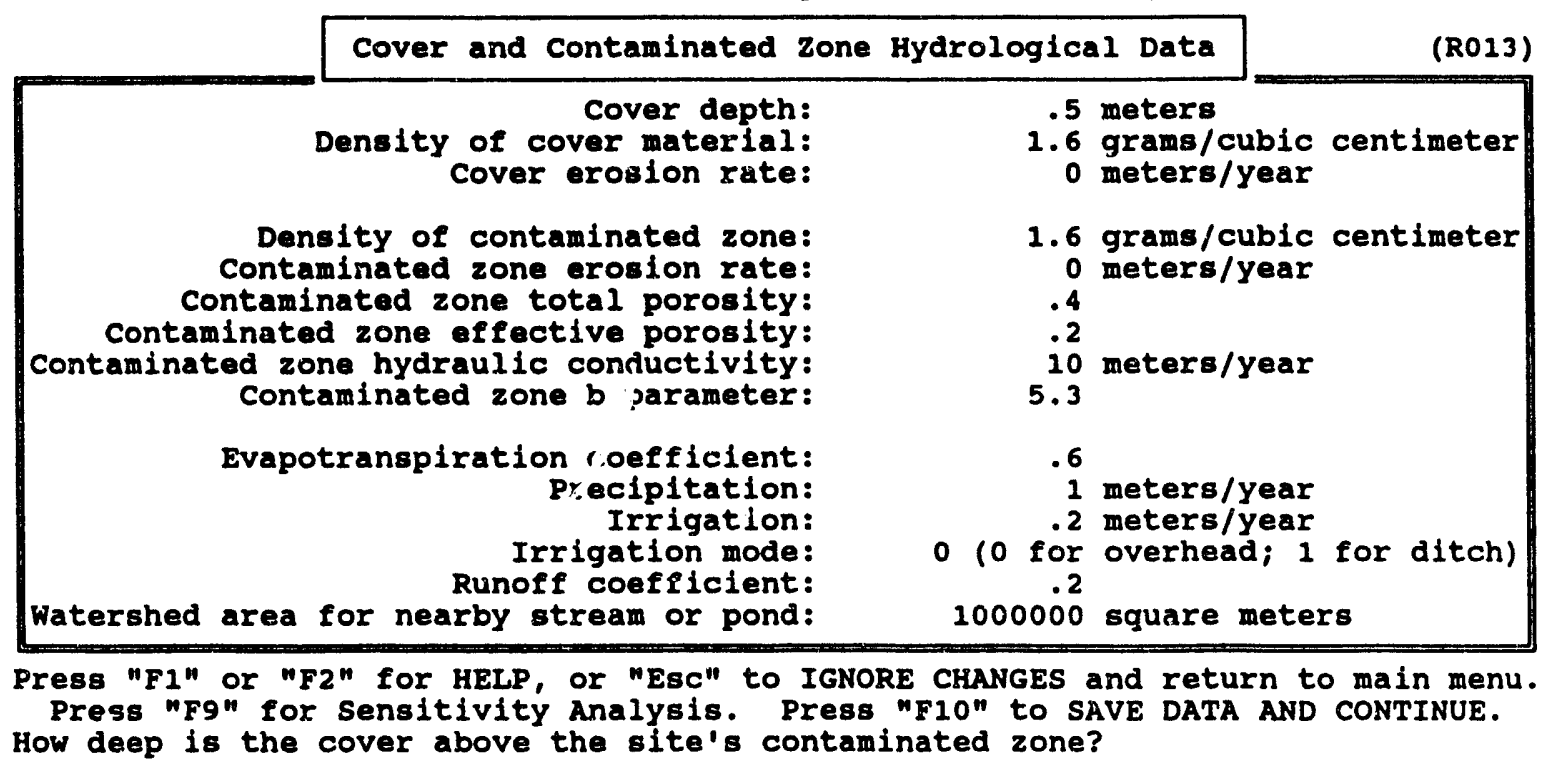

FIGURE 5 Input Form R013 for Cover and Contaminated Zone Hydrological Data 


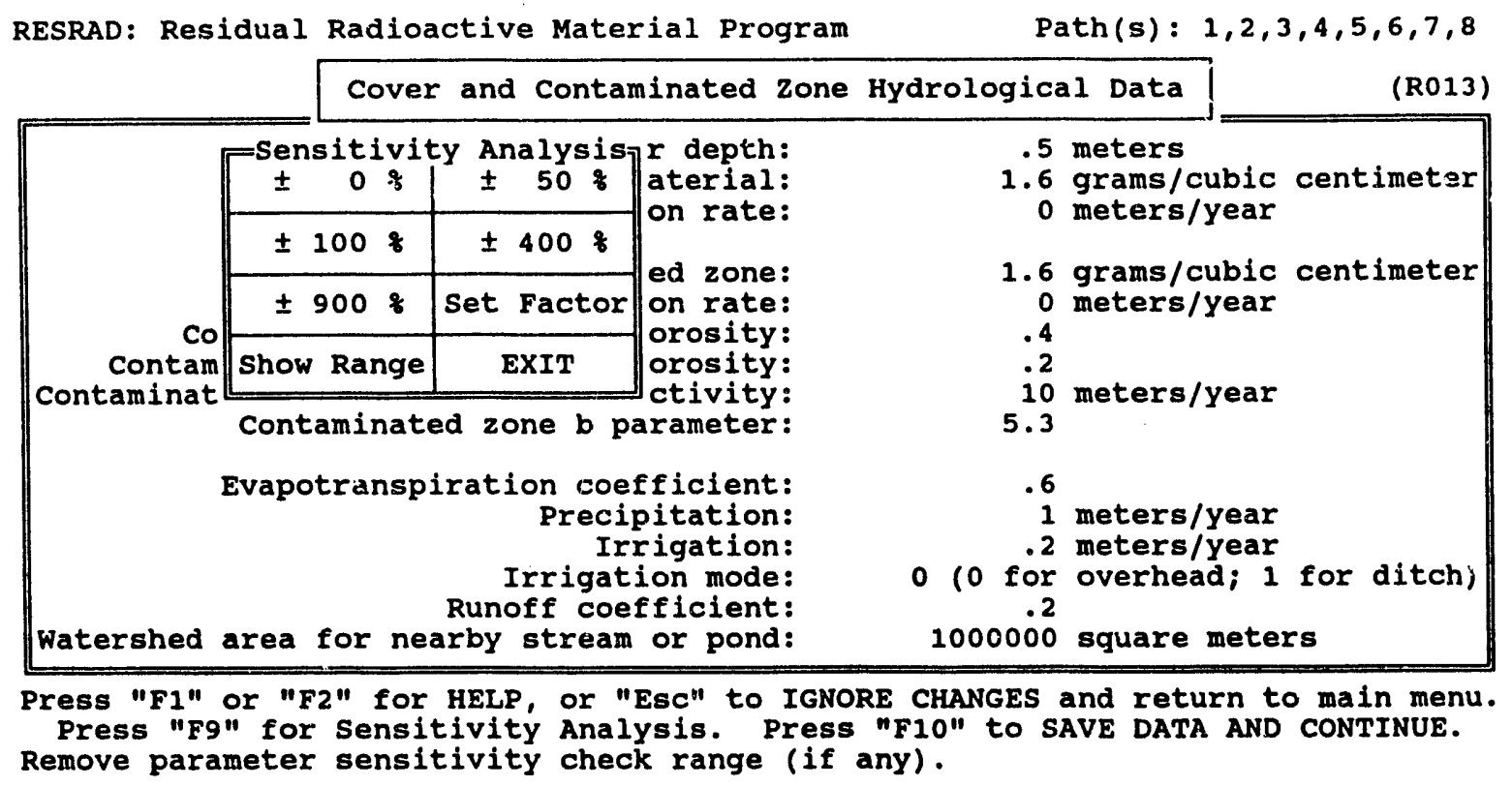

FIGURE 6 Input Form R013 with Sensitivity Analysis Pop-Up Menu

There is always one major calculational cycle in RESRAD. If sensitivity analysis has been requested, there are two more cycles per analysis, up to a maximum of five analyses (CYCLE1, CYCLE2, ... CYCLE9, CYCLE10). The first cycle of each pair changes the parameter being analyzed by the plus percentage set in the input screen; the second cycle decreases the parameter by the specified factor.

The factor $F$, which is used in the sensitivity analysis, is obtained from the screen input range $R$ as a percent and is converted by the following formula: $F=1+0.01 \times R$. Thus, a $400 \%$ range $(R)$ converts to a factor $(F)$ of 5 . The base value of the test parameter is first multiplied by and then divided by $\mathrm{F}$. Results obtained by using three parametric values are plotted in the same figure. Only the value of the parameter being tested is changed in each sensitivity cycle. A maximum of five parameters can be specified for sensitivity analysis in a single run of RESRAD.

\subsection{VIEWING THE SENSITIVITY ANALYSIS RESULTS}

After execution of RESRAD, the calculational results from the sensitivity analysis are not written to the output files SUMMARY.REP and DETAILED.REP as are the results from the base data. Instead, RESRAD displays the sensitivity analysis results in graphics that compare final results (such as TDOSE) calculated from the base parameters and from the perturbed parameters. This way, the user gets a clear picture of the degree of change in the results, which is usually of more concern than the values of the derivatives and sensitivities. The graphics can be output by printers or plotters. Figures 7 through 9 are examples of RESRAD sensitivity analysis graphics. 


\subsection{COMPARING GRAPHIC RESULTS WITH GRESS ENHANCEMENT AND DIRECT PARAMETER PERTURBATION RESULTS}

The sensitivity analysis results obtained by using RESRAD 4.0 with input file SENSE.DAT are shown in Figures 7 through 9. Figure 7 shows the total dose (mrem/yr) from all isotopes summed over all pathways, along with the results of the sensitivity analysis of parameter THICK0, which was derived by multiplying the base value by a factor of 1.5 in the first cycle and by $1 / 1.5$ in the second cycle. The total dose increases as the value of THICKO increases and decreases as the value of THICK0 decreases. This tendency was predictea by the GRESS enhancement and direct parameter perturbation methods, as shown in Tables 1 through 4, by the positive derivatives of THICK0. In Figure 7, DWT0 - the initial depth from the surface to the water table $(5.5 \mathrm{~m})$-- was automatically adjusted by RESRAD when the value of THICKO was changed.

The influence of the parameter PRECIP on the total dose varies from time to time because of a coinplex nonlinear relationship with TDOSE. Increasing the precipitation rate shifts the maximum peak to the left, as can be seen in Figure 8, and decreases the maximum dose of the peak. On the basis of the data provided in Tables 1, 2, and 4, it was predicted that increasing the precipitation rate would decrease the values of TDOSE; the contrary was predicted on the basis of the data in Table 3. Agreement with these predictions is shown in Figure 8, in which the magnitude of TDOSE at 100, 300, and 3000 years decreases as the value of PRECIP increases and vice versa. At 1000 years, however, the magnitude of TDOSE increases as the value of PRECIP increases. It is easier to interpret and understand the sensitivity analysis results in Tables 1 through 4 with the aid of figures such as Figure 8.

The influence of parameter DCACTS(42) on the total dose is shown in Figure 9. Figure 9 shows that DCACTS(42) is very sensitive to the total dose, TDOSE, in the time frame of 300 to 3000 years. The parameter DCACTS(42) is almost insensitive to TDOSE outside this range. (The three curves coincide outside the range.) This result is consistent with the results provided in Tables 1 through 4 in which only Table 3 contains a sensitivity value of -0.4815 at 1000 years. It can be seen from Figure 9 that, at 1000 years, the total dose decreases as the value of DCACTS(42) increases. 
TOTAL DOSE: All Isotor,es and Pathways Summed Sensitivity Analysis: THICKO $\pm 50 \%$

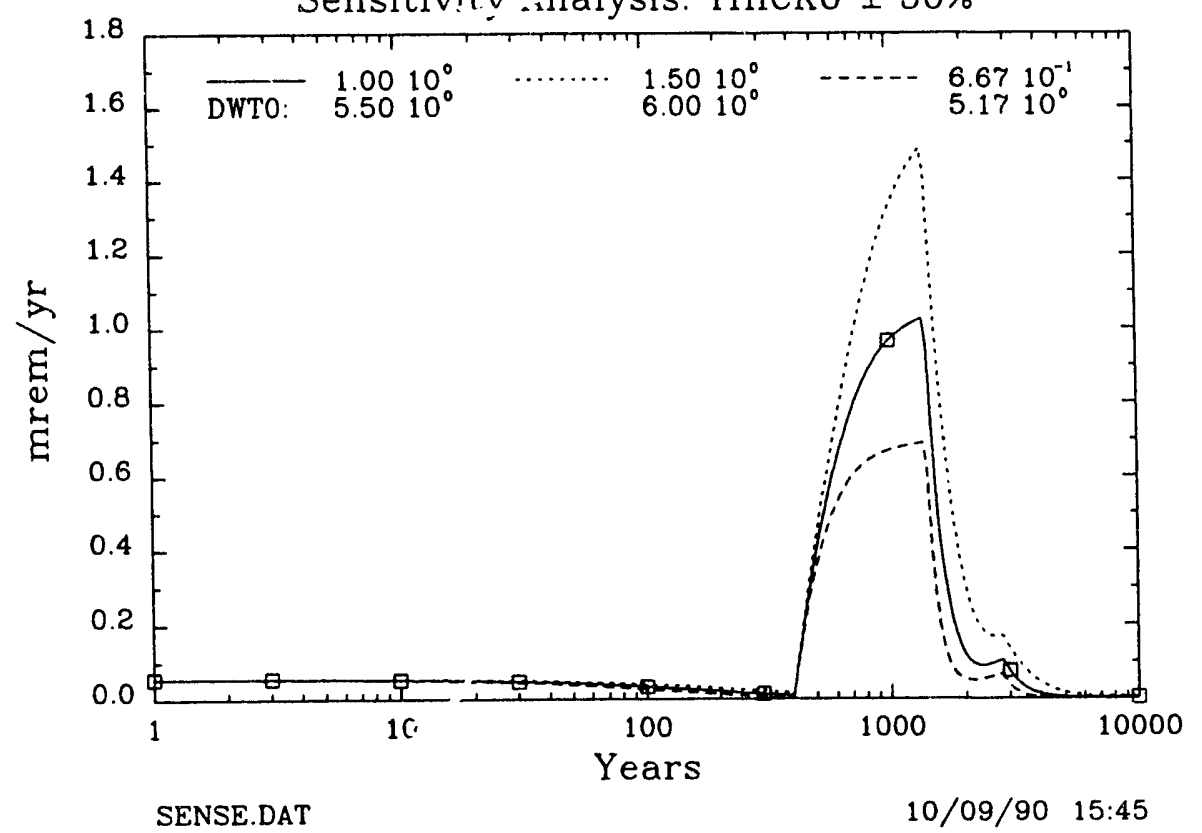

FIGURE 7 Total Dose of All Isotopes Summed over All Pathways with Sensitivity Analysis of THICKO

TOTAL DOSE: All Isotopes and Pathways Summed Sensitivity Analysis: PRECIP $\pm 50 \%$

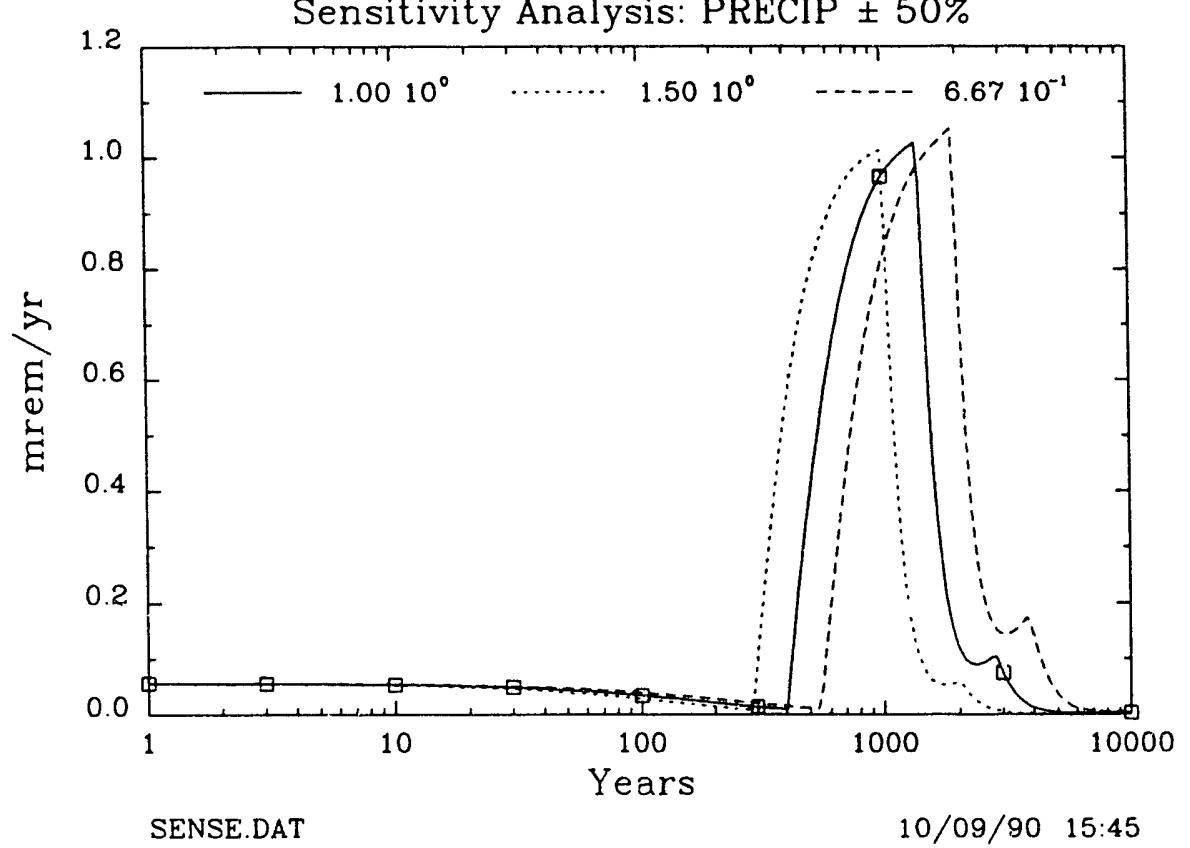

FIGURE 8 Total Dose of All Isotopes Summed over All Pathways with Sensitivity Analysis of PRECIP 
TOTAL DOSE: All Isotopes and Pathways Summed

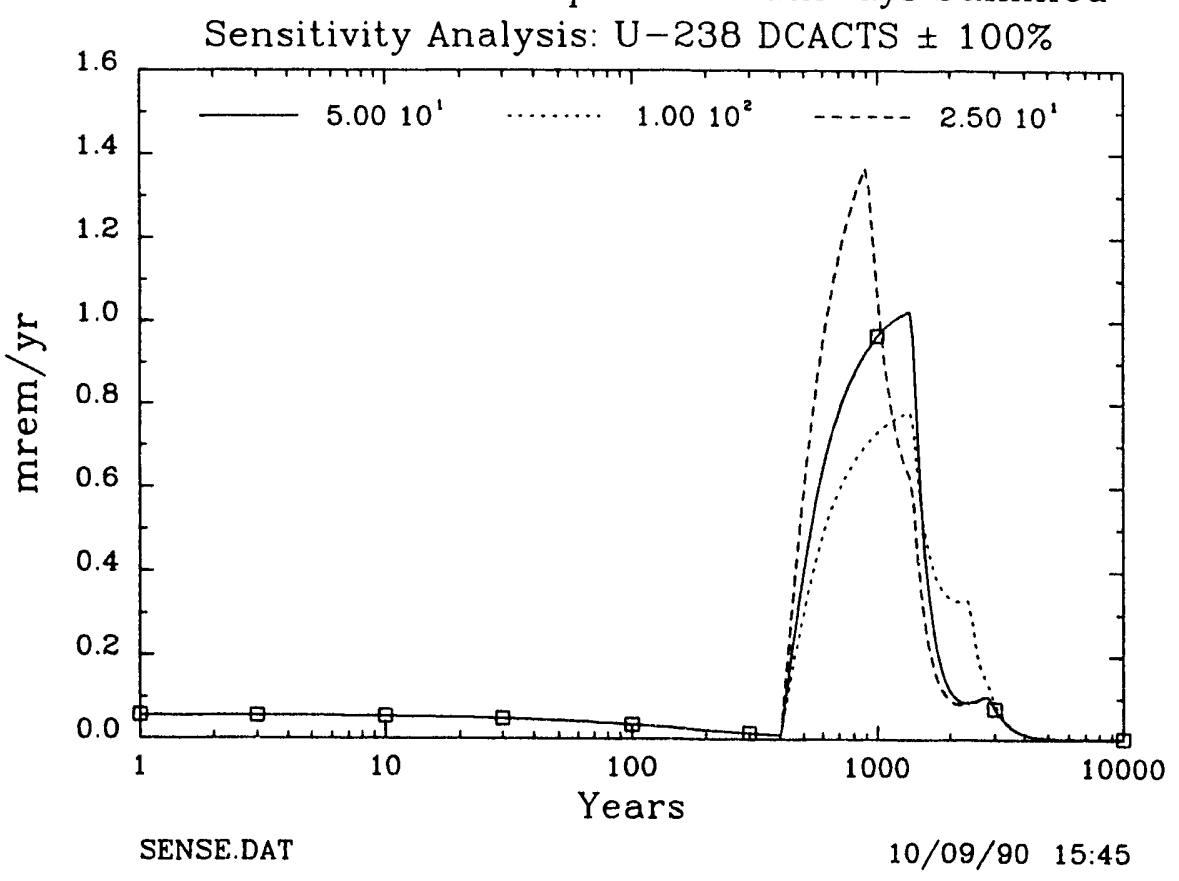

FIGURE 9 Total Dose of All Isotopes Summed over All Pathways with Sensitivity Analysis of DCACTS(42) 


\section{CONCLUSIONS}

A sensitivity analysis of RESRAD code parameters was performed by using GRESS enhancement, direct parameter perturbation, and graphic comparison. Evaluation of these methods indicated that GRESS enhancement has limitations when the response function is discontinuous or a trial-and-error procedure is involved; that direct parameter perturbation is simple but tedious to implement; and that the graphics capability of RESRAD 4.0 is the easiest means of performing sensitivity analyses.

Good agreement in the sensitivities was found between the GRESS enhancement and direct parameter perturbation methods. The analysis showed that parameters such as COVER0, THICK0, DROOT, and DENSCZ, which are associated with the cover material or the contaminated zone, have more influence on the results than parameters of the unsaturated or saturated zone, such as DWIBWT, EPSZ, DENSAQ, and DCACTS, before the breakthrough time of the groundwater contamination. The influence of these parameters changes, however, after the breakthrough time. The parameters EVAPTR, PRECIP, RI, and RUNOFF, which are used to calculate the water infiltration rate, have considerable effect on the results regardless of whether they appear before or after the breakthrough time. The sensitivities of parameters involved in the leaching process have opposite effects on the total dose before and after the rise time as the result of an accumulation-depletion process.

The graphics produced by RESRAD 4.0 reflected the results obtained from the GRESS enhancement and direct parameter perturbation methods. In addition, they provided a direct and easy way to judge the influence of input parameters. To obtain more reliable predictions from the RESRAD code, parameters with high sensitivities should be determined more accurately whenever possible. Accurate sensitivity analysis results can then be used to establish priorities for input data collection. 


\section{REFERENCES}

Gilbert, T.L., et al., 1989, A Manual for Implementing Residual Radioactive Material Guidelines, Argonne National Laboratory Report ANL/ES-160, DOE/CH/8901, June.

Horwedel, J.E., 1990, RSIC Peripheral Shielding Routine Collection -- GRESS 1.0 -- Gradient Enhanced Software System, Oak Ridge National Laboratory Report PSR-31, April.

Press, W.H., et al., 1989, Numerical Recipes - the Art of Scientific Computing, Cambridge University Press, Cambridge, United Kingdom, p. 283. 


\section{APPENDIX A:}

\section{INPUT PARAMETER DEFINITIONS*}

FDW Fraction of drinking water that is contaminated; that is, water that comes from the site. Default $=1$.

FGWDW Fraction of drinking water from groundwater (well). Balance is from surface water (pond). Default $=1$.

FGWIR Fraction of irrigation water from groundwater. Balance is from surface water. Default $=1$; all irrigation water comes from the site.

FO1 Occupancy and shielding factor for external gamma radiation pathways. Default $=0.6$.

FS1 Shape factor for external gamma radiation, dimensionless. Default $=1$.

RUNOFF Runoff coefficient. Default $=0.6$.

$\mathrm{S}(\mathrm{LR}) \quad$ Soil concentration $(\mathrm{pCi} / \mathrm{g}$ ) of principal radionuclide (LR) at initial time.

"For definitions of the remaining parameters, see Appendix B. 


\section{APPENDIX B:}

\section{RESRAD PARAMETERS ELIGIBLE FOR SENSITIVITY ANALYSIS}

The following parameters are eligible for sensitivity analysis when executing RESRAD version 3.121A:

\begin{tabular}{|c|c|}
\hline AREA & Area of the contaminated zone $\left(\mathrm{m}^{2}\right)$ \\
\hline $\mathrm{BCZ}$ & Soil-specific $b$ parameter of contaminated zone \\
\hline $\mathrm{BSZ}$ & Soil-specific b parameter of saturated zone \\
\hline BUZ(i) & Soil-specific $b$ parameter of ith unsaturated zone \\
\hline COVERO & Thickness of clean cover on the contaminated zone (m) \\
\hline DCACTC & $\mathrm{K}_{\mathrm{d}}$ in contaminated zone for indicated isotope $\left(\mathrm{cm}^{3} / \mathrm{g}\right)$ \\
\hline DCACTS & $K_{d}^{u}$ in saturated zone for indicated isotope $\left(\mathrm{cm}^{3} / \mathrm{g}\right)$ \\
\hline DCACTU(i) & $K_{d}$ in ith unsaturated zone for indicated isotope $\left(\mathrm{cm}^{3} / \mathrm{g}\right)$ \\
\hline DENSAQ & Density of saturated zone $\left(\mathrm{g} / \mathrm{cm}^{3}\right)$ \\
\hline DENSCV & Density of cover material $\left(\mathrm{g} / \mathrm{cm}^{3}\right)$ \\
\hline DENSCZ & Density of contaminated zone material $\left(\mathrm{g} / \mathrm{cm}^{3}\right)$ \\
\hline DENSUZ(i) & Density of ith unsaturated zone $\left(\mathrm{g} / \mathrm{cm}^{3}\right)$ \\
\hline DIET(1) & Fruits, nonleafy vegetables, and grain consumption $(\mathrm{kg} / \mathrm{yr})$ \\
\hline $\operatorname{DIET}(2)$ & Leafy vegetable consumption $(\mathrm{kg} / \mathrm{yr})$ \\
\hline DIET(3) & Milk consumption $(\mathrm{L} / \mathrm{yr})$ \\
\hline $\operatorname{DIET}(4)$ & Meat and poultry consumption $(\mathrm{kg} / \mathrm{yr})$ \\
\hline DIET(5) & Fish consumption $(\mathrm{kg} / \mathrm{yr})$ \\
\hline $\operatorname{DIET}(6)$ & Other seafood consumption $(\mathrm{kg} / \mathrm{yr})$ \\
\hline $\mathrm{DM}$ & Depth of soil mixing area $(\mathrm{m})$ \\
\hline DROOT & Depth of roots $(\mathrm{m})$ \\
\hline DWI & Drinking water intake $(\mathrm{L} / \mathrm{yr})$ \\
\hline DWIBWT & Well pump intake depth (m below water table) \\
\hline EPCZ & Effective porosity of contaminated zone \\
\hline EPSZ & Effective porosity of saturated zone \\
\hline EPUZ(i) & Effective porosity of ith unsaturated zone \\
\hline EVAPTR & Evapotranspiration coefficient \\
\hline $\mathrm{H}(\mathrm{i})$ & Thickness of ith unsaturated zone (m) \\
\hline $\mathrm{HCCZ}$ & Hydraulic conductivity of contaminated zone $(\mathrm{m} / \mathrm{yr})$ \\
\hline $\mathrm{HCSZ}$ & Hydraulic conductivity of saturated zone $(\mathrm{m} / \mathrm{yr})$ \\
\hline HCUZ(i) & Hydraulic conductivity of ith unsaturated zone $(\mathrm{m} / \mathrm{yr})$ \\
\hline HGWT & Hydraulic gradient of saturated zone \\
\hline INHALR & Inhalation rate $\left(\mathrm{m}^{3} / \mathrm{yr}\right)$ \\
\hline RI & Irrigation rate $(\mathrm{m} / \mathrm{yr})$ \\
\hline LCZPAQ & Length of contaminated zone parallel to aquifer flow (m) \\
\hline LFI5 & Livestock fodder intake for meat $(\mathrm{kg} / \mathrm{d})$ \\
\hline LFI6 & Livestock fodder intake for milk (kg/d) \\
\hline LM & Dilution length for inhalation of airborne dust (m) \\
\hline LWI5 & Livestock water intake for meat $(\mathrm{L} / \mathrm{d})$ \\
\hline LWI6 & Livestock water intake for milk (L/d) \\
\hline MLFD & Mass loading for foliar deposition $\left(\mathrm{g} / \mathrm{m}^{3}\right)$ \\
\hline
\end{tabular}


MLINH Mass loading for inhalation $\left(\mathrm{g} / \mathrm{m}^{3}\right)$

PRECIP Precipitation $(\mathrm{m} / \mathrm{yr})$

RLEACH Leach rate for indicated isotope $(1 / \mathrm{yr})$

RUNOFF Runoff coefficient

THICK0 Thickness of contaminated zone $(\mathrm{m})$

TPCZ Total porosity of contaminated zone

TPSZ Total porosity of saturated zone

TPUZ(i) Total porosity of ith unsaturated zone

UW

VCV

VCZ

VWT

WAREA

Individual's use of groundwater $\left(\mathrm{m}^{3} / \mathrm{yr}\right)$

Erosion rate of cover material $(\mathrm{m} / \mathrm{yr})$

Erosion rate of contaminated zone $(\mathrm{m} / \mathrm{yr})$

Water table drop rate $(\mathrm{m} / \mathrm{yr})$

Watershed area for nearby stream or pond $\left(\mathrm{m}^{2}\right)$ 


\section{APPENDIX C:}

\section{RESRAD SENSITIVITY INPUT DATA FILE}

The RESRAD input data file SENSE.DAT, which was used for sensitivity analysis, is provided in Figure C.1.

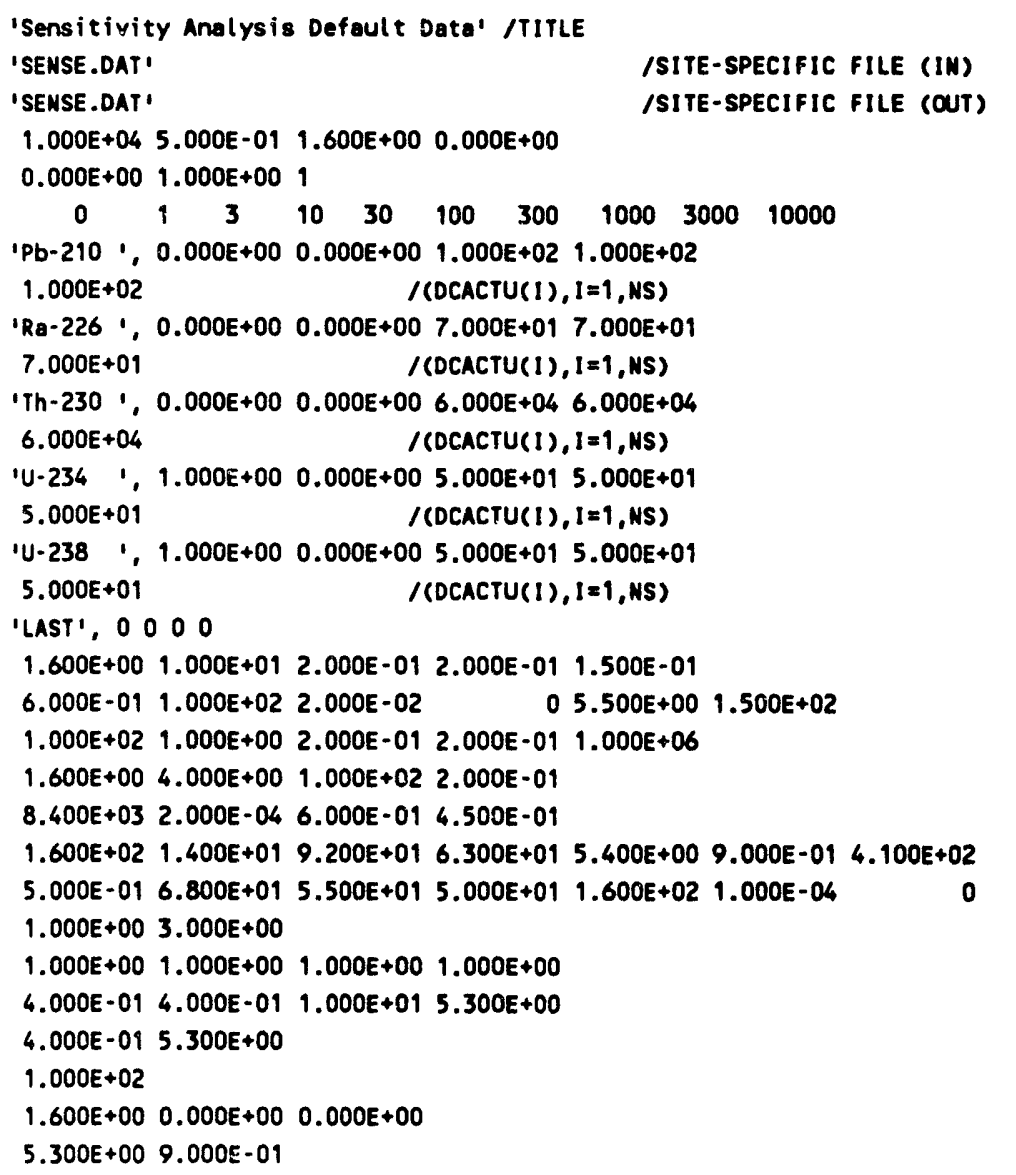

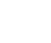




\section{APPENDIX D: \\ TYPICAL RESULTS OF GRESS SENSITIVITY ANALYSIS}

Figure D.1 shows typical sensitivity analysis results obtained by using GRESS. The response function used is the total dose at the eighth year. The parameter sensitivities have been sorted.

\begin{tabular}{|c|c|c|c|}
\hline RESPONSE 22 & ToOSE (8) & 9.650 & $90-01$ \\
\hline MAME & & DERIVATIVE & SENSITIVITY \\
\hline DHIBUT & & $-9.61422 E-02$ & $-9.96277 E-01$ \\
\hline EPS2 & & $-4.80711 E+00$ & $-9.96277 E-01$ \\
\hline DENSAQ & & $-5.97910 E-01$ & $-9.91339 E-01$ \\
\hline TPS2 & & $2.39164 E+00$ & $9.91339 E-01$ \\
\hline DENSCZ & & 5.07441E-01 & $8.41341 E-01$ \\
\hline THICKO & & 8.11325E-01 & $8.40739 E-01$ \\
\hline FDW & & $7.95160 E-01$ & $8.23988 E-01$ \\
\hline DWI & & $1.93942 E-03$ & 8.23988E-01 \\
\hline FGLOH & & $7.87209 E-01$ & $8.15748 E-01$ \\
\hline$s(39)$ & & $4.96128 E-01$ & 5.14115E-01 \\
\hline DCACTS(39) & & $-9.67174 E-03$ & $-5.01119 E-01$ \\
\hline$s(42)$ & & $4.68886 \mathrm{E}-01$ & $4.85885 E-01$ \\
\hline DCACTS(42) & & $-9.29975 E-03$ & $-4.81845 E-01$ \\
\hline EVAPTR & & $-6.80095 E-01$ & $-4.22851 E-01$ \\
\hline PRECIP & & $2.17631 E-01$ & $2.25521 E-01$ \\
\hline RI & & $9.81492 E-01$ & $2.03415 E-01$ \\
\hline FGWIR & & $1.40472 E-01$ & $1.45565 E-01$ \\
\hline$H(15)$ & & $-3.05030 \mathrm{E}-02$ & $-1.26435 E-01$ \\
\hline EPUZ(IS) & & $-6.10059 E-01$ & $-1.26435 E-01$ \\
\hline TPUZ(IS) & & $3.04099 E-01$ & $1.26049 E-01$ \\
\hline DENSUZ(IS) & & $-7.60247 E-02$ & $-1.26049 E-01$ \\
\hline OIET(1) & & $4.85380 E-04$ & 8.04763E-02 \\
\hline DCACTC(39) & & $-1.46561 E-03$ & $-7.59371 E-02$ \\
\hline DCACTC(42) & & $-1.46383 E-03$ & $-7.58452 E-02$ \\
\hline AREA & & $6.81759 E-06$ & $7.06475 E-02$ \\
\hline RUMOFF & & $-2.72038 E-01$ & $-5.63801 \varepsilon-02$ \\
\hline $\operatorname{DIET}(4)$ & & $8.50407 E-04$ & $5.55180 E-02$ \\
\hline DCACTU(39,1) & & $-1.03446 E-03$ & $-5.35981 E-02$ \\
\hline $\operatorname{DCACTU}(42,1)$ & & $-9.94672 E-04$ & $-5.15366 E-02$ \\
\hline LFI5 & & $5.64437 E-04$ & $3.97732 E-02$ \\
\hline $\operatorname{DIET}(2)$ & & $1.71484 E-03$ & $2.48781 E-02$ \\
\hline FGWLW & & $2.34728 E-02$ & $2.43238 E-02$ \\
\hline $\operatorname{DCACTU}(22,1)$ & & $-1.91525 E-04$ & $-1.98469 E-02$ \\
\hline $2 W 15$ & & $3.03878 E-04$ & $1.57448 E-02$ \\
\hline $\operatorname{DIET}(3)$ & & $1.51514 E-04$ & $1.44447 E-02$ \\
\hline
\end{tabular}

FIGURE D.1 GRESS Sensitivity Analysis Results for TDOSE((8) 


\section{APPENDIX E: \\ RESRAD/VAX VERSION SUMMARY REPORT}

The following pages show the RESRAD/VAX Version summary report obtained when the SENSE.DAT input file is used. The table of contents for the summary report is provided below.

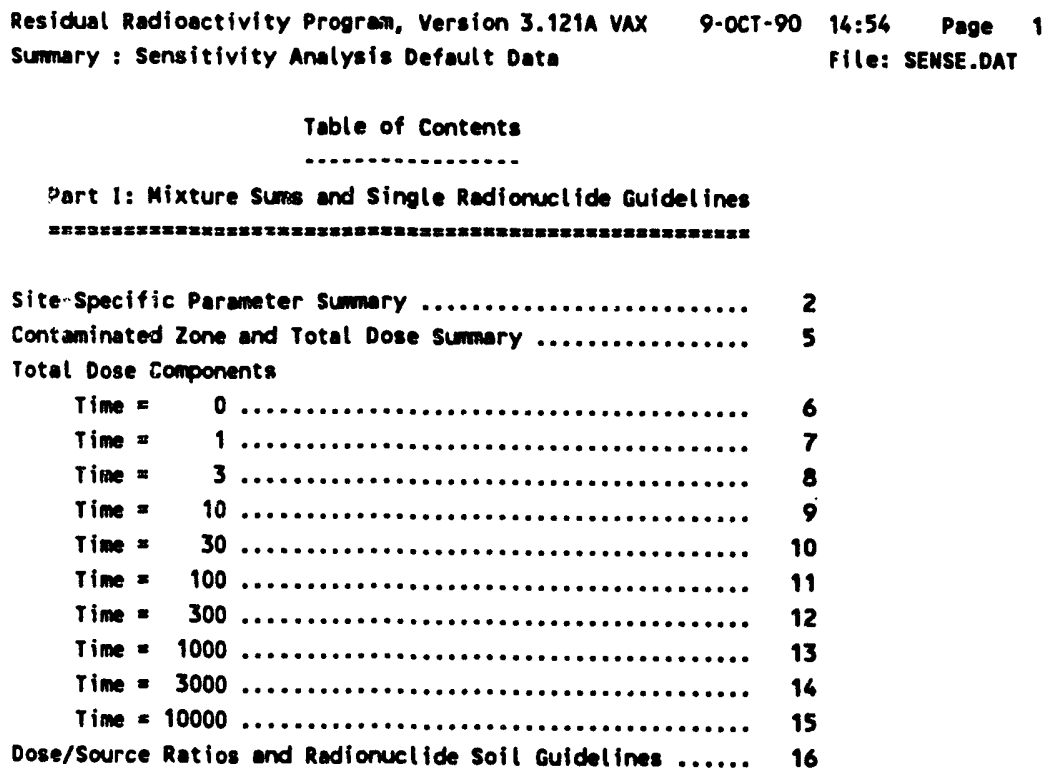


Residual Radioactivity Program, Version 3.121A vaX

9-OCT-90 14:54 Page 2

Sumary : Sensitivity Analys is Defaut t Data

file: SENSE.DAT

Site-Specific Parameter summary

\begin{tabular}{|c|c|c|c|}
\hline Menu | & Parometer & Used & Defoult \\
\hline 2011 & Area of contaminated zone $\left(m^{* * 2}\right)$ & $1.000 E+04$ & $1.000 E+04$ \\
\hline Ro11 & Thickness of conteminated zone $(m)$ & $1.000 E+00$ & $1.000 E+00$ \\
\hline RO11 & length parallel to aquifer flow (m) & $1.000 E+02$ & $1.000 E+02$ \\
\hline Ro11 & Basic radiation dose limit (mrem/yr) & $1.000 E+02$ & $1.000 E+02$ \\
\hline R011 & Tines for calculations (yr) & $1.000 E+00$ & $1.000 E+00$ \\
\hline R011 & Times for calculations (yr) & $3.000 E+00$ & $3.000 E+00$ \\
\hline RO11 & Times for calculations (yr) & $1.000 E+01$ & $1.000 E+01$ \\
\hline R011 & Times for calculations (yr) & $3.000 E+01$ & $3.000 E+01$ \\
\hline RO11 & Times for calculations $(y r)$ & $1.000 E+02$ & $1.000 E+02$ \\
\hline Ro11 & Times for celculations $(y r)$ & $3.000 E+02$ & $3.000 E+02$ \\
\hline R011 & Times for calculations (yr) & $1.000 E+03$ & $1.000 E+03$ \\
\hline R011 & Times for calculations $(y r)$ & $3.000 E+03$ & $3.000 E+03$ \\
\hline 2011 & Times for calculations (yr) & $1.000 E+04$ & $1.000 E+04$ \\
\hline 8012 & Initial principal radionuclide (pci/g): $\quad$-234 & $1.000 E+00$ & $0.000 E+00$ \\
\hline R012 & Initial principal radionuclide (pci/g): $\quad$-238 & $1.000 E+00$ & $0.000 E+00$ \\
\hline Ro13 & Cover depth (m) & $5.000 E-01$ & $5.000 E-01$ \\
\hline R013 & Density of cover material $\left(g / \mathrm{cm}^{m+3}\right)$ & $1.600 E+00$ & $1.600 E+00$ \\
\hline R013 & Cover depth erosion rate $(w / y r)$ & $0.000 E+00$ & $0.000 E+00$ \\
\hline$R 013$ & Density of contaminated $z$ one $\left(g / \mathrm{cm}^{* * 3}\right)$ & $1.600 E+00$ & $1.600 E+00$ \\
\hline 2013 & Conteminated zone erosion rate $(m / y r)$ & $0.000 E+00$ & $0.000 E+00$ \\
\hline $\mathrm{RC13}$ & Conteminated zone total porosity & $4.000 E-01$ & 4.000E-01 \\
\hline R013 & Contaninated zone effective porosity & $2.000 E-01$ & $2.000 E-01$ \\
\hline 2013 & Conteminated zone hydraulic conductivity (m/yr) & $1.000 E+01$ & $1.000 E+01$ \\
\hline R013 & Conteninated zone b paraneter & $5.300 E+00$ & $5.300 E+00$ \\
\hline R013 & Evapotranspiration coefficient & $6.000 E-01$ & $6.000 E-01$ \\
\hline RO13 & Precipitation (m/yr) & $1.000 E+00$ & $1.000 E+00$ \\
\hline R013 & Irrigation $(m / y r)$ & $2.000 E-01$ & $2.000 E-01$ \\
\hline R013 & Irrigation mode & overheed & overhead \\
\hline $\mathbf{R O 1 3}$ & Runoff coefficient & $2.000 E-01$ & $2.000 E-01$ \\
\hline R013 & Watershed area for nearby stream or pond $(n * \star 2)$ & $1.000 E+06$ & $1.000 E+06$ \\
\hline 2014 & Density of saturated zone $\left(\mathrm{g} / \mathrm{cm}^{*} * 3\right)$ & $1.600 E+00$ & $1.600 E+00$ \\
\hline 2014 & Saturated zone total porosity & $4.000 E-01$ & 6.000E-01 \\
\hline R016 & Saturated zone effective porosity & $2.000 E-01$ & 2.000E-01 \\
\hline 2014 & Saturated zone hydraulic corductivity $(w / y r)$ & $1.000 E+02$ & $1.000 E+02$ \\
\hline 2014 & Saturated zone hydraulic gradient & $2.000 E-02$ & $2.000 E-02$ \\
\hline R014 & Distance from surface to water table (m) & $5.500 E+00$ & $5.500 E+00$ \\
\hline R014 & Water table drop rate $(m / y r)$ & $0.000 E+00$ & $0.000 E+00$ \\
\hline 8014 & Well punp intake depth (m below water table) & $1.000 E+09$ & $1.000 \varepsilon+01$ \\
\hline R016 & Model: Mondispersion (ND) or Mass-Balance (MB) & ND & mo \\
\hline R015 & Number of unsaturated zone strata & 1 & 1 \\
\hline $\mathbf{R 0 1 5}$ & Unsat. zone 1, thickness $(m)$ & $4.000 E+00$ & $4.000 E+00$ \\
\hline R015 & Unsat. zone 1, soil density $\left(9 / \mathrm{cm}^{* 4} 3\right)$ & $1.600 E+00$ & $1.600 E+00$ \\
\hline R015 & Unsat. zone 1, total porosity & $4.000 E-01$ & $4.000 E-01$ \\
\hline R015 & Unsat. zone 1, effective porosity & $2.000 E-01$ & $2.000 E-01$ \\
\hline R015 & Unsat. zone 1, soll-specific b paraneter & $5.300 E+00$ & $5.300 E+00$ \\
\hline R015 & Unsat. zone 1, hydraulic conductivity $(m / y r)$ & $1.000 E+02$ & $1.000 E+02$ \\
\hline
\end{tabular}


Residual Radioactivity Progran, Version 3.121A VAX 9-oct-90 14:54 Page 3 Summary : Sensitivity Analysis Default Data File: SENSE.DAT

Site-Specific Parameter Sumary (cont inued)

\begin{tabular}{|c|c|c|c|}
\hline Menu 1 & Parameter & Used & Defoult \\
\hline R016 | & Distribution coefficients for $U-234$ & & \\
\hline$R 016$ i & Contaminated zone $(\mathrm{cm} * \mathbf{3} / \mathrm{g})$ & $5.000 E+01$ & $5.000 E+01$ \\
\hline R016 & Unsaturated zone $1(\mathrm{~cm} * * 3 / 9)$ & $5.000 E+01$ & $5.000 E+01$ \\
\hline 8016 & Saturated zone $(\mathrm{cm} * 3 / \mathrm{g})$ & $5.000 E+01$ & $5.000 E+01$ \\
\hline R016 & Leach rate $(/ y r)$ & $0.000 E+00$ & $0.000 E+00$ \\
\hline R016 & Distribution coefficients for U-238 & & \\
\hline R016 & Contaminated zone $\left(\mathrm{cm}^{* * 3 / g}\right)$ & $5.000 E+01$ & $5.000 E+01$ \\
\hline R016 & Unsaturated zone $1\left(\mathrm{~cm}^{* * 3 / g)}\right.$ & $5.000 E+01$ & $5.000 E+01$ \\
\hline R016 & Saturated zone $\left(\mathrm{cm}^{* * 3 / g}\right)$ & $5.000 E+01$ & $5.000 E+01$ \\
\hline RO16 & Leach rate $(/ y r)$ & $0.000 E+00$ & $0.000 E+00$ \\
\hline$R 016$ & Distribution coefficients for daughter $\mathrm{Pb}-210$ & & \\
\hline R016 & Conteminated zone $\left(\mathrm{cm}^{* * 3} 3 / \mathrm{g}\right)$ & $1.000 E+02$ & $1.000 E+02$ \\
\hline R016 & Unsaturated $20 \mathrm{ne} 1(\mathrm{~cm}+3 / \mathrm{g})$ & $1.000 E+02$ & $1.000 E+02$ \\
\hline R016 & Saturated zone $\left(\mathrm{cm}^{*} * 3 / g\right)$ & $1.000 E+02$ & $1.000 E+02$ \\
\hline R016 & Leach rate $(/ y r)$ & $0.000 E \nrightarrow 00$ & $0.000 E+00$ \\
\hline R016 & Distribution coefficients for daughter Ra-226 & & \\
\hline R016 & Contaminated zone $(\mathrm{cm} * 3 / 9)$ & $7.000 E+0 i$ & $7.000 E+01$ \\
\hline R016 & Unsaturated zone $1(\mathrm{~cm} * 3 / \mathrm{g})$ & $7.000 E+01$ & $7.000 E+01$ \\
\hline R016 & Soturated zone $(\mathrm{cm}+* 3 / \mathrm{g})$ & $7.000 \mathrm{E} \diamond 01$ & $7.000 E+01$ \\
\hline R016 & Leach rate $(/ y r)$ & $0.000 E+00$ & $0.000 E+00$ \\
\hline R016 & Distribution coefficients for daughter Th-230 & & \\
\hline R016 & Contaminated zone $\left(\mathrm{cm}^{*} * 3 / \mathrm{g}\right)$ & $6.000 E+04$ & $6.000 E+04$ \\
\hline R016 & Unsaturated zone $1(\mathrm{~cm} * 3 / \mathrm{g})$ & $6.000 E+04$ & $6.000 E+04$ \\
\hline R016 & Saturated zone $\left(\mathrm{cm}^{*} * 3 / \mathrm{g}\right)$ & $6.000 E+04$ & 6.000E+04 \\
\hline R016 & Leach rate $(/ y r)$ & $0.000 E+00$ & $0.000 E+00$ \\
\hline R017 & Inhalation rate $\left(m^{* * 3 / y r)}\right.$ & $8.400 E+03$ & $8.400 E+03$ \\
\hline R017 & Mass loading for inhalation $\left(g / m^{*+3}\right)$ & $2.000 E-04$ & 2.000E-04 \\
\hline R017 & Uecupancy and shielding factor, external gama & $6.000 E-01$ & $6.000 E-01$ \\
\hline R017 & occupancy factor, inhalotion & 4.500E-01 & 4.500E-01 \\
\hline R017 & Shape factor, external gamma & $1.000 E+00$ & $1.000 E+00$ \\
\hline R017 & Mixing height for airborne dust, inhalation (m) & $3.000 E+00$ & 3.000E+00 \\
\hline RO18 & Fruits, vegetables and orain consumption (kg/yr) & $1.600 E+02$ & $1.600 E+02$ \\
\hline R018 & Leafy vegetable consumption (kg/yr) & $1.400 E+01$ & $1.400 E+01$ \\
\hline$R 018$ & Milk consumption $(L / Y r)$ & $9.200 E+01$ & $9.200 E+01$ \\
\hline R018 & Meat and poultry consumption ( $\mathrm{kg} / \mathrm{yr})$ & $6.300 E+01$ & $6.300 E+01$ \\
\hline R018 & Fish consumption $(\mathrm{kg} / \mathrm{yr})$ & $5.400 E+00$ & $5.400 E+00$ \\
\hline R018 & Other seafood consumpzion (kg/yr) & $9.000 E-01$ & $9.000 E-01$ \\
\hline R018 & Drinking water intake (L/yr) & $4.100 E+02$ & 6. $100 E+02$ \\
\hline$R 018$ & Fraction of drinking water from site & $1.000 E+00$ & $1.000 E+00$ \\
\hline R018 & Fraction of aquatic food from site & $5.000 E-01$ & $5.000 E-01$ \\
\hline R019 & Livestock fodder intake for meat (kg/day) & $6.800 E+01$ & $6.800 E+01$ \\
\hline R019 & Livestock fodder intake for milk (kg/day) & $5.500 E+01$ & 5.500E+01 \\
\hline R019 & Livestock water intake for meat (L/day) & $5.000 E+01$ & $5.000 E+01$ \\
\hline R019 & Livestock water intake for milk (L/day) & $1.600 E+02$ & $1.600 E+02$ \\
\hline R019 & Mass loading for foliar deposition $\left(g / m^{*} 3\right)$ & $1.000 E-04$ & $1.000 E-04$ \\
\hline
\end{tabular}




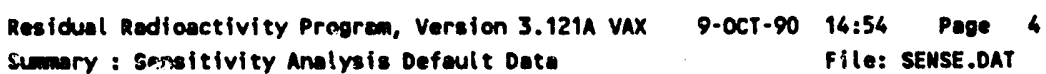

site-Spacific Paremeter sumary (continued)

\begin{tabular}{|c|c|c|c|}
\hline Menu | & Poramezer & Used & Default \\
\hline 2019 & Depth of soil mixing layer (m) & $1.500 E-01$ & $1.500 E-01$ \\
\hline RO19 & Depth of roots $(m)$ & $9.000 E-01$ & $9.000 E-01$ \\
\hline $209 \%$ i & Dririking water fraction from sround water & $1.000 E+D 0$ & $1.000 E+00$ \\
\hline 2019 i & Livestock water frection from ground water & $1.000 E+00$ & $1.000 E+00$ \\
\hline 2019 i & Irrigation fraction from ground water & $1.000 E+00$ & $1.000 E+00$ \\
\hline
\end{tabular}

Residual Redioactivity Progrem, Version 3.121A vax 9-0CT-90 14:56 Poge 5

Sumary : Sencitivity Analysis befoult Data File: SENSE.DAT

\begin{tabular}{|c|c|c|c|}
\hline \multicolumn{2}{|c|}{ Conteminated Zone Dimens: ons } & \multicolumn{2}{|c|}{ Initial Soil Concentrations, $\mathrm{PCi} / \mathrm{g}$} \\
\hline$\ldots . . .-$ & (n) & 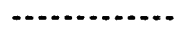 & (n) \\
\hline Aree: & 10000.00 squere meters & U-234 & $1.000 E+00$ \\
\hline Thickness: & 1.00 meters & U-238 & $1.000 E+00$ \\
\hline
\end{tabular}

Total Dose TDOSE( $t$ ), mrem/yr

Basic Rediation Dose Limit = $100 \mathrm{mrew} / \mathrm{r}$

Total Mixture Sum $M(t)=$ Fraction of Basic Dose Liait keceived at Time $(t)$

\begin{tabular}{|c|c|c|c|c|c|c|c|c|c|c|}
\hline$t$ (years): & 0 & 1 & $\mathbf{3}$ & 10 & 30 & 100 & 300 & 1000 & 3000 & 10000 \\
\hline TDOSE $(\tau):$ & $5.635 E-02$ & 5.607E-02 & 5.552E-02 & $5.362 E-02$ & 6.856E-02 & $3.446 E-02$ & $1.390 E-02$ & $9.650 E-01$ & $7.425 E-02$ & $3.062 E-03$ \\
\hline$M(t):$ & $5.635 E-06$ & 5.807E-04 & 5.552E-04 & $5.362 E-04$ & $4.856 E-06$ & $3.446 E-04$ & $1.390 E-04$ & $9.650 E-03$ & $7.425 E-04$ & $3.062 E-05$ \\
\hline
\end{tabular}

Maximun toose(t): $1.029 E+00 \mathrm{mrem} / \mathrm{yr}$ at $t=1606.32: 0.07$ years

Total Dose Contributions TDOSE $(i, p, t)$ for Individual Radionuclides (i) and Pathways $(p)$, mrem/yr

At $t=1406.32$ years

\begin{tabular}{|c|c|c|c|c|c|c|c|c|c|c|c|}
\hline & & Woter Ir & ndependent P & Pathways & & & Water & Dependent $\mathrm{Pz}$ & athways & & \\
\hline Nucl ide & Ground & Dust & Plant & Meat & Milk & Water & Fish & Plant & Meat & $\operatorname{Milk}$ & Total \\
\hline$U-236$ & $9.228 E-06$ & $0.000 E+00$ & $3.290 \mathrm{E}-03$ & $9.754 E-05$ & $1.376 E-06$ & $4.366 E-01$ & $4.545 E-04$ & 6. $118 E-02$ & $2.864 E-02$ & $7.424 E-03$ & 5.377E-01 \\
\hline$U-238$ & $1.182 E-07$ & $0.000 E+00$ & $2.384 E-05$ & $2.766 E-06$ & $3.851 E-07$ & $4.069 E-01$ & $3.217 E-04$ & $4.914 E-02$ & $2.748 E-02$ & $7.170 E-03$ & 4.911E-01 \\
\hline Toto & $347 E-06$ & 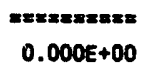 & $\begin{array}{r}x=2 x=x=2 x= \\
3.314 E-03\end{array}$ & $\begin{array}{r}\Rightarrow \approx x=x=z=x= \\
1.003 E-04\end{array}$ & $\begin{array}{r}x=x=x=x=x= \\
1.761 E-06\end{array}$ & $\begin{array}{l}x=z=x==2 \pi= \\
8.435 E-01\end{array}$ & $\begin{array}{l}=x==2=x=== \\
7.762 E-04\end{array}$ & $\begin{array}{r}\Rightarrow=x=z==== \\
\text { 1.103E-01 }\end{array}$ & $5.612 E-02$ & $1.459 \mathrm{E}-02$ & \\
\hline
\end{tabular}

Total Dose Contributions TDOSE $(i, p, t)$ for Individual Radionuclides (i) and Pathways (p), percent

At $t=1406.32$ years

\begin{tabular}{|c|c|c|c|c|c|c|c|c|c|c|c|}
\hline \multirow{3}{*}{ Ruclio- } & \multicolumn{4}{|c|}{ Water Independent Puthways } & \multicolumn{7}{|c|}{ Water Dependent Pathways } \\
\hline & & & & & & & & & & & \\
\hline & iround & Dust & Plant & Meat & Milk & Mater & fish & Plarz & Meat & Milk & \\
\hline & & & & & & & & & & & \\
\hline-236 & 8.9 & $0.000 E+00$ & 3.198E-01 & $9.482 E-03$ & $38 E-04$ & $4.264 E+01$ & $8 E-02$ & & $2.784 E+00$ & & \\
\hline 200 & $1.149 E-05$ & $0.000 E+00$ & $2.318 E-03$ & $2.689 E-06$ & 3.744E-05 & $3.956 E+01$ & 3.127E-02 & 4.TTTE+00 & $2.671 E+00$ & $6.970 E-01$ & $4.774 E+01$ \\
\hline Tot: & $\begin{array}{l}x=x=x=2 x \\
.004 x-04\end{array}$ & 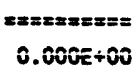 & $\begin{array}{r}x=3 x=2 x=\pi x= \\
3.222 E-0 i\end{array}$ & $\begin{array}{l}:=x===2=x= \\
9.750 E-0 \hat{S}\end{array}$ & 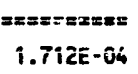 & 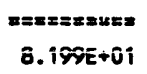 & $\begin{array}{l}=2=\pi x=2=2 x \\
7.545 E-022\end{array}$ & I. OิTZE+טิi & $5.455 E+U \hat{O}$ & i.419E+0̄ô & \\
\hline
\end{tabular}


Residual Radioactivity Program, Version 3.121A VAX 9-oct-90 14:54 Page 6

Sumary : Sensitivity Analysis Default Data File: SEMSE.DAT

Total Dose Contributions $\operatorname{roOSE}(i, p, t)$ for Individual Redionuclides (i) and Pathways $(p), \mathrm{mrem} / \mathrm{yr}$

$$
\text { At } t=0 \text { years }
$$

\begin{tabular}{|c|c|c|c|c|c|c|c|c|c|c|c|}
\hline \multirow[b]{2}{*}{ Nucl ide } & \multicolumn{5}{|c|}{ Water Independent Pathways } & \multicolumn{5}{|c|}{ Water Dependent Pathways } & \multirow[b]{2}{*}{ Total } \\
\hline & Ground & Dust & Plant & Meat & Milk & Water & Fish & Plant & Meat & Milk & \\
\hline & & & & & & & & & & & \\
\hline 234 & $3.751 E-09$ & $0.000 E+00$ & 2.513E-02 & $3.094 E-03$ & 4.385E-04 & $0.000 E+00$ & $0.000 E+00$ & $0.000 E+00$ & $0.000 E+00$ & $0.000 E+00$ & $2.867 E-02$ \\
\hline 238 & $1.245 E-04$ & $0.000 E+00$ & $2.497 \mathrm{E}-02$ & 2.975E-03 & $4.217 E-04$ & $0.000 E+00$ & $0.000 E+00$ & $0.000 E+00$ & $0.000 E+00$ & $0.000 E+00$ & $2.769 \mathrm{E}-02$ \\
\hline $\begin{array}{l}=x==s \\
\text { Total }\end{array}$ & $\begin{array}{r}z=x=x==x= \\
1.245 E-04\end{array}$ & $\begin{array}{r}z=x=x=x== \\
0.000 E+00\end{array}$ & $\begin{array}{r}x=x=x=x=z \\
4.930 E-02\end{array}$ & $\begin{array}{r}=z== \pm==== \\
6.069 E-03\end{array}$ & $\begin{array}{r}z=z===x=== \\
8.602 E-04\end{array}$ & $\begin{array}{r}x==2===2 x \\
0.000 E+00\end{array}$ & $\begin{array}{r}z x=z=z=z=z \\
0.000 E+00\end{array}$ & $\begin{array}{r}==x==x===z \\
0.000 E+00\end{array}$ & $\begin{array}{r}z=x=x=z==x \\
0.000 E+00\end{array}$ & $\begin{array}{r}z=x=z==x== \\
0.000 E+00\end{array}$ & $\begin{array}{r}z==x=x==== \\
5.635 \mathrm{E}-02\end{array}$ \\
\hline
\end{tabular}

Total Dose Contributions $\operatorname{TDOSE}(i, p, t)$ for Individual Radionuclides (i) and Pathways ( $p$ ), percent

$$
\text { At } t=0 \text { years }
$$

\begin{tabular}{|c|c|c|c|c|c|c|c|c|c|c|c|}
\hline \multirow[b]{2}{*}{ Hucl ide } & \multicolumn{5}{|c|}{ Water Independent Pathways } & \multicolumn{5}{|c|}{ Hater Dependent Pathways } & \multirow[b]{2}{*}{ Totat } \\
\hline & Ground & Dust & Plant & Meat & Milk & Water & Fish & $P$ lant & Neat & Milk & \\
\hline & & & & & & & & & & & \\
\hline-234 & $6.656 E-06$ & $0.000 E+00$ & $4.460 E+01$ & $5.490 E+00$ & 7.782E-01 & $2.000 E+00$ & $0.000 E+00$ & $0.000 E+00$ & $0.000 E+00$ & $0.000 E+00$ & $5.087 E+01$ \\
\hline U-238 & $2.209 E-01$ & $0.000 E+00$ & $4.288 E+01$ & $5.279 E+00$ & $7.483 E-01$ & $0.000 E+00$ & $0.000 E+00$ & $0.000 E+00$ & $0.000 E+00$ & $0.000 E+00$ & $4.913 E+01$ \\
\hline$=s=s= \pm=$ & $==x= \pm=x= \pm$ & $z=x=x= \pm x=x$ & $x== \pm== \pm==x$ & $== \pm=x=x==x$ & $== \pm=x= \pm x=x$ & $x= \pm 2=z=z=z$ & $==x== \pm x==$ & $x=z= \pm x=z==$ & $=x==x==x==$ & $==x==x= \pm=z$ & $==x==x====$ \\
\hline Total & $2.210 E-01$ & $0.000 E+00$ & $8.748 E+01$ & $1.077 E+01$ & $1.526 E+00$ & $0.000 E+00$ & $0.000 E+00$ & $0.000 E+00$ & $0.000 E+00$ & $0.000 E+00$ & $1.000 E+02$ \\
\hline
\end{tabular}

Residual Radioactivity Program, Version 3.121A vax 9-oct-90 14:54 Page 7

Summary : Sensitivity Analysis Default Data File: SENSE.DAT

Total Dose Contributions TOOSE(i,p,t) for Individual Radionuclides (i) and Pathways (p), mrem/yr

\begin{tabular}{|c|c|c|c|c|c|c|c|c|c|c|c|}
\hline & & Water In & dependent & pathways & & & Weter D & ependent $\mathrm{Pa}$ & thways & & \\
\hline uelide & Ground & Dust & Plant & Heat & Milk & Water & Fish & & at & Milk & Total \\
\hline & & & . & (n) & & & & & & & \\
\hline-234 & 3.827E-09 & $0.000 E+00$ & 2.501E-02 & $3.079 E-03$ & $4.364 E-04$ & $0.000 E+00$ & $0.000 E+00$ & $0.000 E+00$ & $0.000 E+00$ & $0.000 E \nrightarrow 00$ & $2.852 E-02$ \\
\hline-238 & $1.239 E-04$ & $0.000 E+00$ & $2.405 E-02$ & $2.960 E-03$ & $4.196 E-04$ & $0.000 E+00$ & $0.000 E+00$ & $0.000 E+00$ & $0.000 E+00$ & $0.000 E+00$ & $2.755 E-02$ \\
\hline $\begin{aligned} &=:== \\
& \text { Tota }\end{aligned}$ & $\begin{array}{l}===x==== \\
1.239 E-04\end{array}$ & $\begin{array}{r}z==z==x==x \\
0.000 E+00\end{array}$ & $\begin{array}{r}==\pi=x=2=x= \\
4.906 \mathrm{E}-02\end{array}$ & $\begin{array}{r}z==x=m=x= \\
6.039 E-03\end{array}$ & $\begin{array}{r}=x=x=m= \\
8.559 E-04\end{array}$ & $\begin{array}{r}z=\pi x=x=\pi=x \\
0.000 E+00\end{array}$ & $\begin{array}{r}x=x x=x=x=x \\
0.000 E+00\end{array}$ & $\begin{array}{r}x=x=\pi=x=m= \\
0.000 E+00\end{array}$ & $\begin{array}{l}m=====x=== \\
0.000 E+00\end{array}$ & $\begin{array}{r}z=x=z=x=z= \\
0,000 E+00\end{array}$ & \\
\hline
\end{tabular}

$$
\text { At } t=1 \text { years }
$$

\begin{tabular}{|c|c|c|c|c|c|c|c|c|c|c|c|}
\hline & & Water In & dependent & Pathways & & & Water D & pendent $\mathrm{Pa}$ & & & \\
\hline lucl ide & Ground & Dust & Plant & Meat & & & sh & & Meat & Milk & Total \\
\hline & $\cdots$ & $\ldots$ & $\ldots$ & & & & & & & & \\
\hline 1.234 & $6.824 E-06$ & $0.000 E+00$ & $4.460 E+01$ & $5.490 E+00$ & 7.782E-01 & $0.000 E+00$ & $0.000 E+00$ & $0.000 E+00$ & $0.000 E+00$ & $0.000 E+00$ & $5.087 E+01$ \\
\hline$J-238$ & $2.209 E-01$ & $0.000 E+00$ & $4.288 E+01$ & $5.279 E+00$ & $7.482 E-01$ & $0.000 E+00$ & $0.000 E+00$ & $0.000 E+00$ & $0.000 E+00$ & $0.000 E+00$ & $4.913 E+01$ \\
\hline rota & $209 E-01$ & 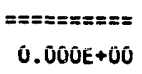 & 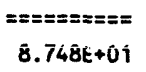 & 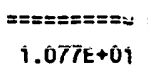 & $\begin{array}{r}========== \\
1.526 E+00\end{array}$ & $\begin{aligned} &=x=2= \pm= \pm== \\
& 0.000 E+00\end{aligned}$ & $\begin{array}{l}z==z====== \\
0.000 E+00\end{array}$ & $\begin{array}{r}z====2=z=== \\
0.000 E+00\end{array}$ & $0.000 E+00$ & $\begin{array}{r}=x===z=x= \\
0.000 E+00\end{array}$ & \\
\hline
\end{tabular}

Total Dose Contributions TOOSE(i,p,t) far Individual Radionuclides (i) and Pathways ( $p$ ), percent

At $t=1$ years 
Residual Radioactivity Progrem, Version 3.121A VAX 9-0CT-90 14:54 Page 8

Sumary : Sensitivity Analysis Default Data File: SENSE.DAT

Total Dose Contributions TDOSE(i,p,t) for Individual Radionuclides (i) and Pathways ( $p$ ), mrem/yr

At $t=3$ years

\begin{tabular}{|c|c|c|c|c|c|c|c|c|c|c|c|}
\hline \multirow[b]{2}{*}{ Nuclide } & \multicolumn{5}{|c|}{ Water Independent Pathways } & \multicolumn{5}{|c|}{ Water Dependent Pathways } & \multirow[b]{2}{*}{ Total } \\
\hline & Ground & Dust & Plant & Meat & Milk & Water & Fish & Plant & Meat & Milk & \\
\hline & & & & & & & & & & & \\
\hline 234 & $4.538 E-09$ & $0.000 E+00$ & $2.476 E-02$ & $3.048 E-03$ & $4.320 E-0.4$ & $0.000 E+00$ & $0.000 E+00$ & $0.000 E+00$ & $0.000 E+00$ & $0.000 E+00$ & $2.824 E-02$ \\
\hline 238 & $1.227 \mathrm{E}-04$ & $0.000 E+00$ & $2.381 E-02$ & 2.931E-03 & $4.154 E-04$ & $0.000 E+00$ & $0.000 E+00$ & $0.000 E+00$ & $0.000 E+00$ & $0.000 E+00$ & $2.728 E-02$ \\
\hline$z=z=x=$ & 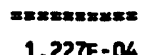 & $=2 x=2 x=2=2 x$ & 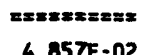 & $=x=1=x=x=x$ & $=x x=== \pm=x$ & $x=2 x=z=x=$ & $x=2=8==8=$ & mx=ex=s==x & I=s=s=s=s= & "x=se=s=s & $z== \pm=2==$ \\
\hline Total & $1.227 E-04$ & $0.000 E+00$ & $4.05 / E-02$ & $5.979 E-03$ & $8.474 E-04$ & $0.000 E+\infty 0$ & $0.000 E+00$ & $0.000 E+00$ & $0.000 E+00$ & $0.000 E+00$ & 5.552E-02 \\
\hline
\end{tabular}

Total Dose Contributions ToOSE $(i, p, t)$ for Individual Radionuclides (i) and Pathways (p), percent

At $t=3$ years

Water Independent Pathways

\begin{tabular}{|c|c|c|c|c|c|c|c|c|c|c|c|}
\hline Nucl ide & Ground & Dust & Plent & Meat & Milk & Water & Fish & Plant & Meat & Milk & Total \\
\hline & - n-then & $\cdots$ & & $\cdots$ & -... & & & $\cdots$ & & & \\
\hline$U-234$ & $8.173 E-06$ & $0.000 E+00$ & $6.660 E+01$ & $5.491 E+00$ & 7.781E-01 & $0.000 E+00$ & $0.000 E+00$ & $0.000 E+00$ & $0.000 E+00$ & $0.000 E+00$ & $5.087 E+01$ \\
\hline$U \cdot 238$ & $2.209 E-01$ & $0.000 E+00$ & $4.288 E+01$ & $5.279 E+00$ & 7.482E-01 & $0.000 E+00$ & $0.000 E+00$ & $0.000 E+00$ & $0.000 E+00$ & $0.000 E+00$ & $4.913 E+01$ \\
\hline $\begin{array}{r}\text { mensze: } \\
\text { Total }\end{array}$ & $2.209 \varepsilon-01$ & $0.000 E+00$ & 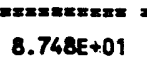 & $\begin{array}{r}x=x z=x=E=x \\
1.077 E+01\end{array}$ & $\begin{array}{r}=2 x=2 x=2 x=2 \\
1.526 E+00\end{array}$ & $\begin{array}{l}z=x=x=x=x= \\
0.000 E+00\end{array}$ & $\begin{array}{l}z=x x=z=x== \\
0.000 E+00\end{array}$ & $\begin{array}{l}z==x===x=2 \\
0.000 E+00\end{array}$ & $\begin{array}{l}z=====x=x= \\
0.000 E+00\end{array}$ & $\begin{array}{r}I==z==x==2 \\
0.000 E+00\end{array}$ & $\begin{array}{r}z======== \\
1.000 E+02\end{array}$ \\
\hline
\end{tabular}

Residual Redioactivity Program, Version 3.121A Vax $9-0 \mathrm{CT}-90 \quad 14: 54 \quad$ Page 9 Sumary : Sensitivity Anslysis Defoult Data File: SENSE.DAT

Total Dose Contributions TOOSE(i,p,t) for Individual Radionuclides (i) and Pathways (p), arem/yr

$$
\text { At } t=10 \text { years }
$$

\begin{tabular}{|c|c|c|c|c|c|c|c|c|c|c|c|}
\hline & & Woter Ir & ndependent $P$ & Pathways & & & Water & Dependent $\mathrm{Pz}$ & thways & & \\
\hline lucl ide & Ground & Dust & Ptant & Meat & & Water & Fish & Plant & Meat & Milk & Total \\
\hline & & & & & & & & & & & \\
\hline $1 \cdot 234$ & 7SE-08 & $0.000 E+00$ & 2. 792E-02 & 2.945E-03 & $.172 E-04$ & $0.000 E+00$ & $0.000 E+00$ & $0.000 z+00$ & $0.000 E+00$ & $0.000 E+00$ & $2.728 E-02$ \\
\hline$u-238$ & $1.185 E-04$ & $0.000 E+00$ & $2.299 E-02$ & 2. B31E-03 & 4.012E-04 & $0.000 E+00$ & $0.000 E+00$ & $0.000 E+00$ & $0.000 E+00$ & $0.000 E+00$ & $2.634 E-02$ \\
\hline Totel & $\begin{array}{l}m=x=2 x=3= \\
1.185 E-04\end{array}$ & 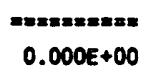 & $\begin{array}{r}m=x=2 x=10= \\
6.691 E-02\end{array}$ & 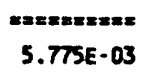 & $\begin{array}{r}m=x=2=x=2 x \\
8.184 E-04\end{array}$ & $\begin{array}{r}z x=z=x=2=x \\
0.000 E+00\end{array}$ & $\begin{array}{r}y=x=x=x= \pm x \\
0.000 E+00\end{array}$ & $0.000 E+00$ & $0.000 E+00$ & $0.000 E+00$ & \\
\hline
\end{tabular}

Total Dose Contributions TOOSE (i,p,t) for Individual Radionuclides (i) and Pathways (p), percent

$$
\text { At } t=10 \text { years }
$$

\begin{tabular}{|c|c|c|c|c|c|c|c|c|c|c|c|}
\hline & & Water In & ndependent f & Iays & & & Water & Dependent P & hways & & \\
\hline Nucl ide & Ground & Dust & Plant & Neat & $k$ & Wat & Fish & Plant & Heat & Milk & \\
\hline & $\ldots$ & & & & & & & & & & \\
\hline$U-234$ & 2.376E-05 & $0.000 E+00$ & $4.461 E+01$ & $5.491 E+00$ & $7.780 E-01$ & $0.000 E+00$ & $0.000 E+00$ & $0.000 E+00$ & $0.000 E+00$ & $0.000 E+00$ & $5.087 E+01$ \\
\hline$U-238$ & $2.209 E-01$ & $0.000 E+00$ & $4.288 E+01$ & $5.278 E+00$ & $7.481 E-01$ & $0.000 E+00$ & $0.000 E+00$ & $0.000 E+00$ & $0.000 E+00$ & $0.000 E+00$ & $4.913 E+01$ \\
\hline Tote & $\begin{array}{r}z=x=\pi x=2 x= \\
2.209 E-01\end{array}$ & $\begin{array}{r}z=2=\pi \varepsilon=2 x= \\
0.000 E+00\end{array}$ & $\begin{array}{r}x=x=2=2 x=2 \\
8.748 E+01\end{array}$ & 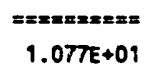 & $\begin{array}{r}x=x=z=x=x \\
1.526 E+00\end{array}$ & $\begin{array}{r}=m=x=2= \pm=x \\
0.000 E+00\end{array}$ & $\begin{array}{r}=\pi=x=2 x==\pi \\
0.000 E+00\end{array}$ & $0.000 E+00$ & 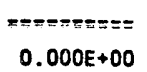 & $0.000 E+00$ & 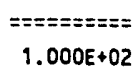 \\
\hline
\end{tabular}


Residual Redioactivity Program, Version 3.121A VAX $\quad 9-0 \mathrm{CT}-90 \quad 14: 54 \quad$ Page 10 Sumary : Sensitivity Analysis Default Data File: SENSE.DAT

Total Dose Coneributions $\operatorname{TDOSE}(i, p, t)$ for Individual Radionuclides (i) and Pathways $(p), \mathrm{mrem} / \mathrm{yr}$

At $t=30$ years

\begin{tabular}{|c|c|c|c|c|c|c|c|c|c|c|c|}
\hline \multirow{3}{*}{$\begin{array}{l}\text { Radio- } \\
\text { Nucl ide }\end{array}$} & \multicolumn{5}{|c|}{ Water Independent Pathways } & \multicolumn{5}{|c|}{ Water Dependent Pathways } & \multirow[b]{3}{*}{ Total } \\
\hline & & & & & 西 & & -0 & $-2 \mathrm{sec}$ & 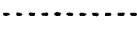 & & \\
\hline & Ground & Dust & Plant & Meat & Milk & Water & Fish & Plant & Meat & Milk & \\
\hline & 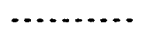 & & & & & & & & & & \\
\hline & $8.094 E-08$ & $0.000 E+00$ & $2.167 E-02$ & $2.667 E-03$ & $3.776 E-04$ & $0.000 E+00$ & $0.000 E+00$ & $0.000 E+00$ & $0.000 E+00$ & $0.000 E+00$ & $2.472 E-02$ \\
\hline$U \cdot 238$ & $1.072 E-04$ & $0.000 E+00$ & $2.081 E-02$ & $2.562 E-03$ & $3.632 E-04$ & $0.000 E+00$ & $0.000 E+00$ & $0.000 E+00$ & $0.000 E+00$ & $0.000 E+00$ & $2.385 E-02$ \\
\hline & & & 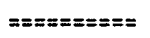 & & =ニニニ=ニニニニ== & & 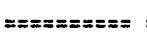 & 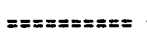 & 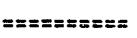 & $n=x==x=x==$ & \\
\hline Total & $1.073 E-04$ & $0.000 E+00$ & $4.249 E-02$ & $5.230 E-03$ & $7.408 E-04$ & $0.000 E+00$ & $0.000 E+00$ & $0.000 E+00$ & $0.000 E+00$ & $0.000 E+00$ & $4.856 E-02$ \\
\hline
\end{tabular}

Total Dose Contributions TDOSE $(i, p, t)$ for Individual Radiomuclides (i) and Pathways ( $p$ ), percent

$$
\text { At } t=30 \text { years }
$$

\begin{tabular}{|c|c|c|c|c|c|c|c|c|c|c|c|}
\hline & & Water In & ndependent P & athways & & & Water D & Dependent $\mathrm{Pa}$ & thways & & \\
\hline uclide & & Dust & Plant & Meat & ilk & & Fish & & leat & Milk & $0 t a t$ \\
\hline & & & & & & & & & & & \\
\hline 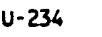 & $.667 \mathrm{E}-04$ & $0.000 E+00$ & $4.463 E+01$ & $5.492 E+00$ & $7.776 E-01$ & $0.000 E+00$ & $0.000 E+00$ & $0.000 E+00$ & $0.000 E+00$ & $0.000 E+00$ & $5.090 E+01$ \\
\hline-238 & $2.208 E-01$ & $0.000 E+00$ & $4.286 E+01$ & $5.276 E+00$ & $7.478 E-01$ & $0.000 E+00$ & $0.000 E+00$ & $0.000 E+00$ & $0.000 E+00$ & $0.000 E+00$ & $4.910 E+01$ \\
\hline $\begin{array}{r}=x==z \\
\text { Total }\end{array}$ & $\begin{array}{l}========= \\
2.210 E-01\end{array}$ & $\begin{array}{r}=====\pi==== \\
\\
0.000 E+00\end{array}$ & $\begin{array}{r}========== \\
\text { 8. } 749 E+01\end{array}$ & $\begin{aligned} &=x==x=== \\
& 1.077 \mathrm{E}+01\end{aligned}$ & $\begin{aligned}= & =\pi=\pi==\pi= \\
& 1.525 E+00\end{aligned}$ & $\begin{aligned} &== \pm=\pi=\pi=x= \\
& 0.000 E+00\end{aligned}$ & $\begin{array}{r}= \pm= \pm=E=z== \\
0.000 E+00\end{array}$ & $\begin{array}{r}========= \\
0.000 E+00\end{array}$ & $\begin{array}{r}===== \pm== \pm= \\
0.000 ₹+00\end{array}$ & $\begin{aligned} &== \pm== \pm=== \pm \\
& 0.000 E+00\end{aligned}$ & $\begin{array}{l}===== \pm==== \\
1.000 E+02\end{array}$ \\
\hline
\end{tabular}

Residual Radioactivity Program, Version 3.121A VAX 9-OCT-90 $14: 54$ Page 11

Sumary : Sensitivity Analysis Default Data File: SENSE.DAT

Total Dose Contributions $\operatorname{rDOSE}(i, p, t)$ for Individual Radionuclides (i) and Pathways $(p)$, mrem/yr

At $t=100$ years

Water Independent Pathways

\begin{tabular}{|c|c|c|c|c|c|}
\hline Muclide & Ground & Dust & Plant & Meat & \\
\hline & $\cdots$ & $\ldots$ & $\cdots+\ldots$ & . & \\
\hline$U \cdot 234$ & $7.061 E-07$ & $0.000 E+00$ & $1.547 E-02$ & $1.891 E-03$ & 2.665 \\
\hline$u-238$ & $7.567 E-05$ & $0.000 E+00$ & $1.469 E-02$ & $1.809 E-03$ & $2.563 E$ \\
\hline & $=-1$ & & 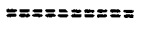 & $==\pi=\pi==\pi=$ & \\
\hline Total & 7.637 E- 05 & $0.000 E+00$ & $3.017 E-02$ & $3.699 E-03$ & \\
\hline
\end{tabular}

Water Dependent Pathways

\begin{tabular}{|c|c|c|c|c|c|}
\hline Waier & Fish & Plant & Meat & Milk & Total \\
\hline n. & $\cdots$ & $\ldots$ & 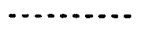 & $\cdots$ & \\
\hline $0.000 E+00$ & $0.000 E+00$ & $0.000 E+00$ & $0.000 E+00$ & $0.000 E+00$ & $1.763 E-02$ \\
\hline $0.000 E+00$ & $0.000 E+00$ & $0.000 E+00$ & $0.000 E+00$ & $0.000 E+00$ & $1.683 E-02$ \\
\hline $\begin{array}{r}z=x==z==== \\
0.000 E+00\end{array}$ & $\begin{array}{r}z======== \\
0.000 E+00\end{array}$ & $\begin{array}{l}z=z==x==z \\
0.000 E+00\end{array}$ & $\begin{array}{r}z=x=3 x=z= \\
0.000 E+00\end{array}$ & $\begin{array}{r}z=x=x=x x=x \\
0.000 E+00\end{array}$ & $\begin{array}{l}x==x==x= \\
3,446 \mathrm{E}-02\end{array}$ \\
\hline
\end{tabular}

Total Dose Contributions $\operatorname{TDOSE}(i, p, t)$ for Individual Radionuclides (i) and Pathways (p), percent

At $t=100$ years

Water Independent Pathways

\begin{tabular}{|c|c|c|c|c|c|}
\hline Vuclide & Ground & Dust & Plant & Heat & Milk \\
\hline$\cdots$ & $\cdots$ & (n) & $\cdots$ & $\ldots$ & $\ldots \ldots$ \\
\hline$U-234$ & $2.049 E-03$ & $0.000 E+00$ & $4.490 E+01$ & $5.486 E+00$ & 7.732E-01 \\
\hline$U-238$ & $2.196 E-01$ & $0.000 E+00$ & $4.263 E+01$ & $5.248 E+00$ & $7.438 E-01$ \\
\hline$==x==x=$ & $===\pi=z=z==$ & $==$ = = = = = = & $========0=$ & 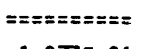 & $====x=5==$ \\
\hline Total & $2.216 E-01$ & $0.000 E+00$ & 8. $753 E+01$ & $1.073 E+01$ & $1.517 E+00$ \\
\hline
\end{tabular}

Water Dependent Pathways

\begin{tabular}{|c|c|c|c|c|c|}
\hline Hater & Fish & Plant & Meat & Milk & Total \\
\hline 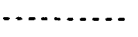 & $\cdots$ & $\ldots$ & $\cdots$ & $\cdots$ & n. \\
\hline $0.000 E+00$ & $0.000 E+00$ & $0.000 E+00$ & $0.000 E+00$ & $0.000 E+00$ & $5.116 E+01$ \\
\hline $0.000 E+00$ & $0.000 E+00$ & $0.000 E+00$ & $0.000 E+00$ & $0.000 E+00$ & $4.884 E+01$ \\
\hline$==\pi= \pm$ & 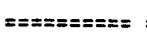 & 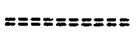 & 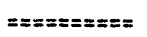 & 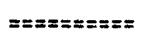 & 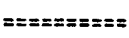 \\
\hline $0.000 E+00$ & $0.000 E+00$ & $0.000 E+00$ & $0.000 E+00$ & $0.000 E+00$ & $1.000 E+02$ \\
\hline
\end{tabular}


Residual Radioactivity Program, Version 3.1214 VaX 9-0CT-90 14:54 Page 12 Summary : Sensitivity Anolysis Default Dato File: SENSE.DAT

Total Does Contribution TDOSE(i,p,t) for Individual Radionuclides (i) and Pathways ( $p$ ), mrem/yr

At $t=300$ years

\begin{tabular}{|c|c|c|c|c|c|c|c|c|c|c|c|}
\hline \multirow[b]{2}{*}{ Muclide } & \multicolumn{5}{|c|}{ Water Independent Pathwoys } & \multicolumn{5}{|c|}{ Water Dependent Pathways } & \multirow[b]{2}{*}{ Total } \\
\hline & Ground & Dust & Plent & Meot & Milk & Water & Fisk & Plant & Meat & Milk & \\
\hline & & & 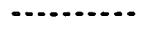 & . & $\cdots$ & $\cdots$ & & & 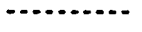 & .. & \\
\hline 234 & $3.652 E-06$ & $0.000 E+00$ & $6.840 E-03$ & 7.351E-04 & $.869 E-05$ & $0.000 E+00$ & $0.000 E+00$ & $0.000 E+00$ & $0.000 E+00$ & $0.000 E+00$ & $7.677 E-03$ \\
\hline-238 & 2.795E-05 & $0.000 E+00$ & $3.429 E-03$ & $6.683 E-046$ & $9.473 E-05$ & $0.000 E+00$ & $0.000 E+00$ & $0.000 E+00$ & $0.000 E+00$ & $0.000 E+00$ & $6.220 E-03$ \\
\hline & $x=2 x=x=12 x$ & $m=x=2 x=$ & zexzexz=zx & szexz=zez= & $=x=\pi=x=$ & 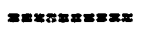 & 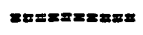 & 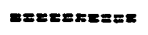 & $\varepsilon=2=2 x= \pm E x$ & $z= \pm x=2 x=2$ & $x=2= \pm=2=x$ \\
\hline Total & $3.160 E-05$ & $0.000 E+00$ & $1.227 \mathrm{E}-02$ & $1.403 E-03$ & $1.934 E-04$ & $0.000 E+00$ & $0.000 E+00$ & $0.000 E+00$ & $0.000 E+00$ & $0.000 E+00$ & $1.390 E-02$ \\
\hline
\end{tabular}

Total Dose Contributions TDOSE $(i, p, t)$ for Individual Radionuclides (i) and Pathways (p), percent

At $t=300$ years

\begin{tabular}{|c|c|c|c|c|c|c|c|c|c|c|c|}
\hline \multirow[b]{2}{*}{ Muclide } & \multicolumn{5}{|c|}{ Water Independent Pathways } & \multicolumn{5}{|c|}{ Water Dependent Pathways } & \multirow[b]{2}{*}{ Total } \\
\hline & Ground & Dust & Plant & Meat & Milk & Water & Fish & Plant & Meat & Milk & \\
\hline & & & & & & & & & & & \\
\hline-234 & $2.628 E-02$ & $0.000 E+00$ & $4.922 E+01$ & $5.289 E+00$ & 7.102E-01 & $0.000 E+00$ & $0.000 E+00$ & $0.000 E+00$ & $0.000 E+00$ & $0.000 E+00$ & $5.524 E+09$ \\
\hline$u-238$ & 2.011E-01 & $0.000 E+00$ & $3.907 E+01$ & $4.809 E+00$ & $6.816 E-01$ & $0.000 E+00$ & $0.000 E+00$ & $0.000 E+00$ & $0.000 E+00$ & $0.000 E+00$ & $4.476 E+01$ \\
\hline$x=2 x=$ & $== \pm x=x= \pm 3 z$ & $z=2=3 \times 5=3 x$ & 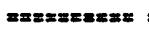 & 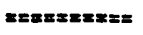 & zx=m & $=:= \pm=2=3$ & & $=x=x=2$ & $y=z x=z=z=3$ & szx=z=zz=z & \\
\hline Total & 2.274E-01 & $0.000 E+00$ & $8.828 E+01$ & $1.010 E+01$ & $1.392 E+\infty 0$ & $0.000 E+00$ & $0.000 E+00$ & $0.000 E+00$ & $0.000 E+00$ & $0.0 n O E+00$ & $1.000 E+02$ \\
\hline
\end{tabular}

Residual Rediaactivity Program, Version 3.121A VAX 9-0CT-90 14:54 Page 13

Summary : Sensitivity Analysis Default Data File: SEMSE.DAT

Total Dose Contributions $\operatorname{TDOSE}(i, p, t)$ for Individual Redionuclides (i) and Pathways ( $p$ ), arem/yr

At $t=1000$ years

Water Independent Pathway

\begin{tabular}{|c|c|c|c|c|c|c|c|c|c|c|c|}
\hline \multirow[b]{2}{*}{ Muclide } & \multicolumn{4}{|c|}{ Water Independent Pathwoys } & \multicolumn{7}{|c|}{ Water Dependent Pathways } \\
\hline & Ground & Dust & Plent & Meat & Milk & Water & Fish & Plant & Meat & Milk & Total \\
\hline 234 & & & $3.2755-03$ & $11100-0$ & & & & & & & \\
\hline 254 & $0.190-\infty$ & 0.000 t + 00 & $3.2 \mathrm{BSE}-03$ & $1.719 E-04$ & $3.934 E-06$ & 4.067E-01 & $3.539 E-04$ & 5. $333 E-02$ & 2.721E-02 & $7.088 E-03$ & 4.961E-01 \\
\hline & 8.601E-07 & $0.000 E+00$ & $1.681 E-04$ & 2.055E-05 & 2.907E-06 & $3.886 E-01$ & $3.070 E-04$ & $4.690 E-02$ & $2.623 E-02$ & $6.844 E-03$ & $4.6895-01$ \\
\hline Total & $\begin{array}{l}x=2 x=2=x=2 \\
9.658 E-06\end{array}$ & $\begin{array}{l}x=2 x=2 x=x \\
0.000 E+00\end{array}$ & $\begin{array}{r}=x=\pi=x=x=x \\
3.443 E-03\end{array}$ & $\begin{array}{r}z=2=2 x=2 x= \\
1.325 E-04\end{array}$ & 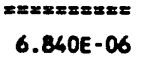 & $\begin{aligned} x=x=2=x=2 \\
7.952 E-01\end{aligned}$ & $\begin{array}{c}z=x=x=2 x z \\
6.609 E-04\end{array}$ & $\begin{array}{r}=2 x=2==== \\
9.823 E-02\end{array}$ & $\begin{array}{l}z==x==x x= \\
5.344 E-02\end{array}$ & $\begin{array}{r}=x=z=x==x= \\
1.393 \mathrm{E}-02\end{array}$ & $\begin{array}{l}=z=\varepsilon=z=z=z \\
9.650 \mathrm{E}-01\end{array}$ \\
\hline
\end{tabular}

Total Dose Contributions TDOSE(i,p,t) for Individual Radionuclides (i) and Pathways ( $p$ ), percent

At $t=1000$ years

\begin{tabular}{|c|c|c|c|c|c|c|c|c|c|c|c|}
\hline \multirow[b]{2}{*}{ Mucl ide } & \multicolumn{5}{|c|}{ Water Independent Pathways } & \multicolumn{5}{|c|}{ Hater Dependent Pathways } & \multirow[b]{2}{*}{ Total } \\
\hline & Ground & Dust & Plent & Meat & Milk & Water & Fish & Plant & Meat & Milk & \\
\hline 234 & $9.117 E-04$ & $0.000 E+00$ & 3.394E-01 & $1.160 E-02$ & $4.076 E-04$ & $4.215 E+01$ & $3.667 E-02$ & $5.319 E+00$ & $2.820 E+00$ & $7.345 E-01$ & $5.141 E+01$ \\
\hline 238 & B.913E-05 & $0.000 E+00$ & $1.742 E-02$ & $2.129 E-03$ & $3.012 E-04$ & $4.025 E+01$ & $3.181 E-02$ & $4.860 E+00$ & $2.718 E+00$ & $7.092 E-01$ & $4.859 E+01$ \\
\hline $\begin{array}{r}=x x=x= \\
\text { Total }\end{array}$ & $\begin{array}{r}x=x=x=z=x \\
1.001 E-03\end{array}$ & $\begin{array}{r}z=x=x=z=z= \\
0.000 E+00\end{array}$ & $\begin{array}{r}x=2=x=x=x= \\
3.568 E-01\end{array}$ & $\begin{array}{r}=z=2 x=2= \\
1.373 E-02\end{array}$ & $\begin{array}{r}x x==== \pm=x= \\
7.088 E-04\end{array}$ & $\begin{array}{r}==E= \pm x=x=x \\
8.240 E+01\end{array}$ & $\begin{array}{c}=z= \pm==2=x= \\
6.848 E-02\end{array}$ & $\begin{array}{r}======== \\
1.018 E+01\end{array}$ & $\begin{array}{r}===z== \pm=2 z \\
5.538 E+00\end{array}$ & $\begin{array}{r}==z==x==2= \\
1.444 E+00\end{array}$ & $\begin{array}{r}z== \pm===== \\
1.000 E+02\end{array}$ \\
\hline
\end{tabular}


Residual Radioactivity Program, Version 3.121A VaX 9-0CT-90 14:54 Page 14

Sumbary : Sensitivity Analysis Default Data File: SENSE.DAT

Total Dose Contributions $\operatorname{TDOSE}(i, p, t)$ for Individual Radionuclides (i) and Pathways $(p)$, mrem/yr

At $t=3000$ years

\begin{tabular}{|c|c|c|c|c|c|c|c|c|c|c|c|}
\hline & & Water In & ndependent $P$ & athways & & & Water D & Dependent $\mathrm{Pa}$ & thways & & \\
\hline Nuclide & Ground & Dust & Plant & Meat & Milk & Water & Fish & Plant & Meat & Milk & Total \\
\hline$U-234$ & $9.173 E-06$ & $0.000 E+00$ & $3.252 E-03$ & $9.405 E-05$ & $9.754 E-07$ & $3.925 E-02$ & $2.999 E-04$ & $3.021 E-02$ & $7.237 E-04$ & $1.849 E-05$ & $7.386 E-02$ \\
\hline$U-238$ & $5.265 E-09$ & $0.000 E+00$ & $1.860 E-06$ & $5.454 E-08$ & $6.934 E-10$ & $2.666 E-04$ & $1.040 E-06$ & $1.107 E-04$ & $1.206 E-05$ & $2.622 E-06$ & $3.949 E-04$ \\
\hline $\begin{aligned}=: z= \pm= & = \\
& \text { Total }\end{aligned}$ & $\begin{array}{r}====2===3= \\
9.178 E-06\end{array}$ & $\begin{array}{r}x=x=x=x=x= \\
0.000 E+00\end{array}$ & $\begin{array}{l}x=====x== \\
3.254 \mathrm{E}-03\end{array}$ & $\begin{array}{r}=x==x=x=x= \\
9.411 E-05\end{array}$ & $\begin{array}{r}x==x=x==x= \\
9.761 E-07\end{array}$ & $\begin{array}{r}===8== \pm==8 \\
3.952 E-02\end{array}$ & $\begin{array}{l}z=x==x==5 x \\
3.009 E-04\end{array}$ & $\begin{array}{l}x==x==x== \\
3.032 E-02\end{array}$ & $\begin{array}{r}==x=== \pm==8 \\
7.358 E-04\end{array}$ & $\begin{array}{r}==\pi==z=== \\
2.111 E-05\end{array}$ & $\begin{array}{r}======== \\
7.425 E-02\end{array}$ \\
\hline
\end{tabular}

Total Dose Contributions TDOSE( $i, p, t)$ for Individual Radionuclides (i) and Pathways (p), percent

At $t=3000$ years

\begin{tabular}{|c|c|c|c|c|c|c|c|c|c|c|c|}
\hline \multirow[b]{2}{*}{ Nuclide } & \multicolumn{5}{|c|}{ Water Independent Pathways } & \multicolumn{5}{|c|}{ Water Dependent Pathways } & \multirow[b]{2}{*}{ Total } \\
\hline & Ground & Dust & Plant & Meat & Milk & Water & Fish & Plant & Meat & Milk & \\
\hline & & & & & & & & & . & . & \\
\hline .234 & $1.235 E-02$ & $0.000 E+00$ & $4.379 E+00$ & $1.267 \mathrm{E}-01$ & 1.314E-03 & $5.286 E+01$ & $4.039 E-01$ & $4.069 E+01$ & $9.747 E-01$ & $2.490 E-02$ & $9.947 E+01$ \\
\hline 238 & $7.090 E-06$ & $0.000 E+00$ & $2.505 E-03$ & $7.345 E-05$ & $9.339 E-07$ & $3.590 E-01$ & $1.400 E-03$ & $1.491 E-01$ & $1.625 E-02$ & $3.532 E-03$ & $5.319 E-01$ \\
\hline$== \pm= \pm$ & =:==s=s=s= & $=x=x=x=x$ & $z=z=\geq=z=\pi=$ & $=x=x=x=x=$ & $=x=x x=x x=8$ & $x=x=x= \pm x==$ & $=x=x=z=x==$ & $==2=2=3 x=3$ & $x=z===x=x=$ & z=:=x=:=es= & \\
\hline Total & $1.236 E-02$ & $0.000 E+00$ & $4.382 E+00$ & $1.267 \mathrm{E}-01$ & $1.315 E-03$ & $5.322 E+01$ & $4.053 E-01$ & $4.084 E+01$ & $9.909 E-01$ & $2.843 E-02$ & $1.000 E+02$ \\
\hline
\end{tabular}

Residual Radioactivity Program, Version 3.121A VAx 9-0CT-90 14:54 Page 15

Sumary : Sensitivity Analysis Default Data File: SENSE.DAT

Total Dose Contributions ToOSE(i,p,t) for Individual Radionuclides (i) and Pathways $(p)$, mrem/yr

At $t=10000$ years

\begin{tabular}{|c|c|c|c|c|c|c|c|c|c|c|c|}
\hline \multirow[b]{2}{*}{ Nuclide } & \multicolumn{5}{|c|}{ Water Independent Pathways } & \multicolumn{5}{|c|}{ Water Dependent Pathways } & \multirow[b]{2}{*}{ Total } \\
\hline & Ground & Dust & Plant & Meat & Milk & Water & Fish & Plont & Meat & Milk & \\
\hline 234 & $8.366 E-06$ & $0.000 E+00$ & $2065 \mathrm{~F}-03$ & & & & & & & & \\
\hline-238 & $4.765 E-09$ & $0.000 E+00$ & $\begin{array}{l}2.965 E-03 \\
1.689 E-06\end{array}$ & $8.577 E-05$ & 8.894E-07 & $5.752 E \cdot 09$ & $4.419 E-11$ & 4.479E-09 & $1.053 E-10$ & $2.028 E-12$ & $3.060 E-03$ \\
\hline$:=== \pm$ & $=x=x=x==x$ & 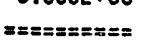 & $=x===2=x=$ & 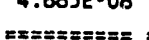 & $3.066 \mathrm{E} \cdot 10$ & $6.860 E-11$ & $5.271 \mathrm{E}-13$ & $5.342 E-11$ & $1.256 E-12$ & $2.418 E-14$ & $1.743 E-06$ \\
\hline Total & $8.370 E-06$ & $0.000 E+00$ & $2.967 E-03$ & $8.582 E-05$ & $8.899 E-07$ & $5.821 E-09$ & $4.472 E-11$ & $4.532 E-09$ & $1.065 E-10$ & $\begin{array}{r}x=x=x=x= \\
2.052 E-12\end{array}$ & $\begin{array}{l}z==== \pm== \\
3.062 E-03\end{array}$ \\
\hline
\end{tabular}

Total Dose Contributions TOOSE(i,p,t) for Individual Radionuclides (i) and Pathways (p), percent

At $t=10000$ years

Water Independent Pathways

\begin{tabular}{|c|c|c|c|c|c|}
\hline Nuclide & Ground & Dust & Plant & Meat & Milk \\
\hline$\ldots$ & $\cdots$ & 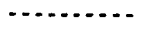 & - & $\cdots \cdots$ & \\
\hline$U \cdot 234$ & $2.732 E-01$ & $0.000 E+00$ & $9.684 E+01$ & $2.801 E+00$ & $2.904 E-02$ \\
\hline$U-238$ & $1.556 E-04$ & $0.000 E+00$ & $5.516 E-02$ & $1.595 E-03$ & $1.654 E-05$ \\
\hline & $===1=====$ & $z=x=x==0$ & z==s=="=x= & =x=m=x==x= & $=z=x==8=0$ \\
\hline rotal & 2.7S3E-01 & $0.000 E+00$ & $9.689 E+01$ & $2.803 E+00$ & $2.906 E-02$ \\
\hline
\end{tabular}

Water Dependent Pathways

\begin{tabular}{|c|c|c|c|c|c|}
\hline Water & Fish & Plant & Meat & Milk & Total \\
\hline 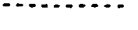 & (n) & , & $\cdots$ & $\cdots$ & \\
\hline $1.879 E-04$ & $1.443 \mathrm{E}-06$ & $1.463 E-04$ & $3.438 E-06$ & $6.622 E-08$ & $9.994 E+01$ \\
\hline $2.240 E-06$ & $1.721 E-08$ & $1.744 E-06$ & 4. 100E-08 & $7.898 E-10$ & $5.693 E-02$ \\
\hline
\end{tabular}


Residual Redioactivity Progrm, Version 3.121A vax 9-oct-90 14:56 Page 16

Sumary : Sensitivity Analysis Defeult Date File: SENSE.DAT

Dose/source Ratios sunmed Over All Pathuays, (mren/yr)/(pCi/g)

\begin{tabular}{|c|c|c|c|c|c|c|c|c|c|c|}
\hline Muclic & & & & & & & & & & \\
\hline (i) & $t=$ & 1 & 3 & 10 & 30 & 100 & 300 & 1000 & 3000 & 10000 \\
\hline -....... & - & 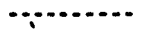 & & & & & & & & \\
\hline$v-234$ & $2.867 E-02$ & $2.852 E-02$ & $2.824 E-02$ & $2.728 E-02$ & 2.472E-02 & $1.763 E-02$ & 7.677E-03 & 4.961E-01 & $7.3866-02$ & $3.060 E-03$ \\
\hline $\begin{array}{l}\text { U-238 } \\
\text { Exz=z=z }\end{array}$ & 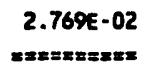 & 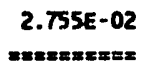 & 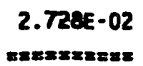 & 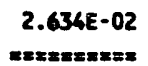 & 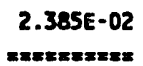 & 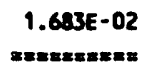 & 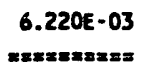 & 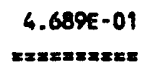 & $\begin{array}{r}3.949 \mathrm{E}-06 \\
=2= \pm=2 x=2\end{array}$ & $\begin{array}{r}1.743 E-06 \\
=x=3=x=2 x\end{array}$ \\
\hline
\end{tabular}

Single Redionuclide soil quidelines $G(i, t)$ in $\mathrm{PCi} / \mathrm{g}$

Esesic Rediation Dose Limit = $100 \mathrm{mrem} / \mathrm{yr}$

\begin{tabular}{|c|c|c|c|c|c|c|c|c|c|c|}
\hline clide & & & & & & & & & & \\
\hline (i) & $t=$ & 1 & 3 & 10 & 30 & 100 & 300 & 1000 & 3000 & \\
\hline & 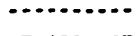 & 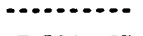 & & & & & & & & \\
\hline 234 & $488 E+03$ & $3.506 E+03$ & $3.541 E+03$ & $3.666 E+03$ & $4.066 E+03$ & $5.671 E+03$ & $1.303 E+06$ & $2.016 E+02$ & $1.354 E+03$ & $3.2000 t+1$ \\
\hline 238 & $3.612 E+03$ & $3.630 E+03$ & $3.666 E+03$ & $3.796 E+03$ & $4.193 E+03$ & $5.941 E+03$ & $1.608 E+04$ & $2.133 E+02$ & $2.532 E+05$ & $-3.360 E+0$ \\
\hline$=z$ & 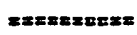 & 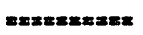 & 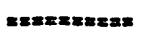 & 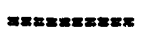 & 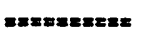 & 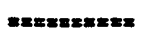 & 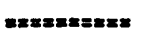 & 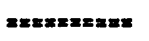 & 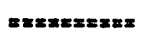 & \\
\hline
\end{tabular}

-At specific activity linit

\begin{tabular}{|c|c|c|c|c|c|c|}
\hline $\begin{array}{l}\text { Nucl ide } \\
\text { (i) }\end{array}$ & $\begin{array}{l}\text { Initiol } \\
\text { pCi/g }\end{array}$ & $\begin{array}{c}\text { tmin } \\
\text { (years) }\end{array}$ & DSR $(1, t$ tmin $)$ & $\begin{array}{c}G(i, t \operatorname{tmin}) \\
(p c i / g)\end{array}$ & DSR $\left(i, t_{\max } x\right)$ & $\begin{array}{l}G(i, t \tan x) \\
(p c i / g)\end{array}$ \\
\hline 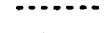 & 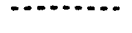 & . & 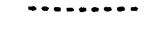 & ................ & 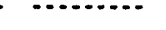 & 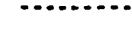 \\
\hline U.234 & $1.000 E+00$ & $1406.28: 0.07$ & 5.37TE-01 & $1.860 E+02$ & 2 5.377E-01 & $1.860 E+02$ \\
\hline $\begin{array}{l}\text { U-238 } \\
=2=x=8 z\end{array}$ & $\begin{array}{l}1.000 E+00 \\
=x=m=x=x=\end{array}$ & 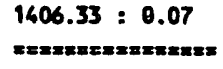 & $\begin{array}{l}\text { 4.911E-01 } \\
=x=x=x=x=3\end{array}$ & 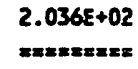 & 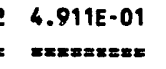 & $\begin{array}{l}2.036 E+02 \\
==2 \times x \times==\end{array}$ \\
\hline
\end{tabular}




\section{DISTRIBUTION FOR ANL/EAIS-3}

\section{Internal}
ANL Contract Copy
K.S. Macal (6)
ANL Patent Department
C. Yu (375)
ANL Technical Publications Services (3)

\section{External}

U.S. Department of Energy Office of Scientific and Technical Information (12) Manager, U.S. Department of Energy Chicago Operations Office ANL Libraries 

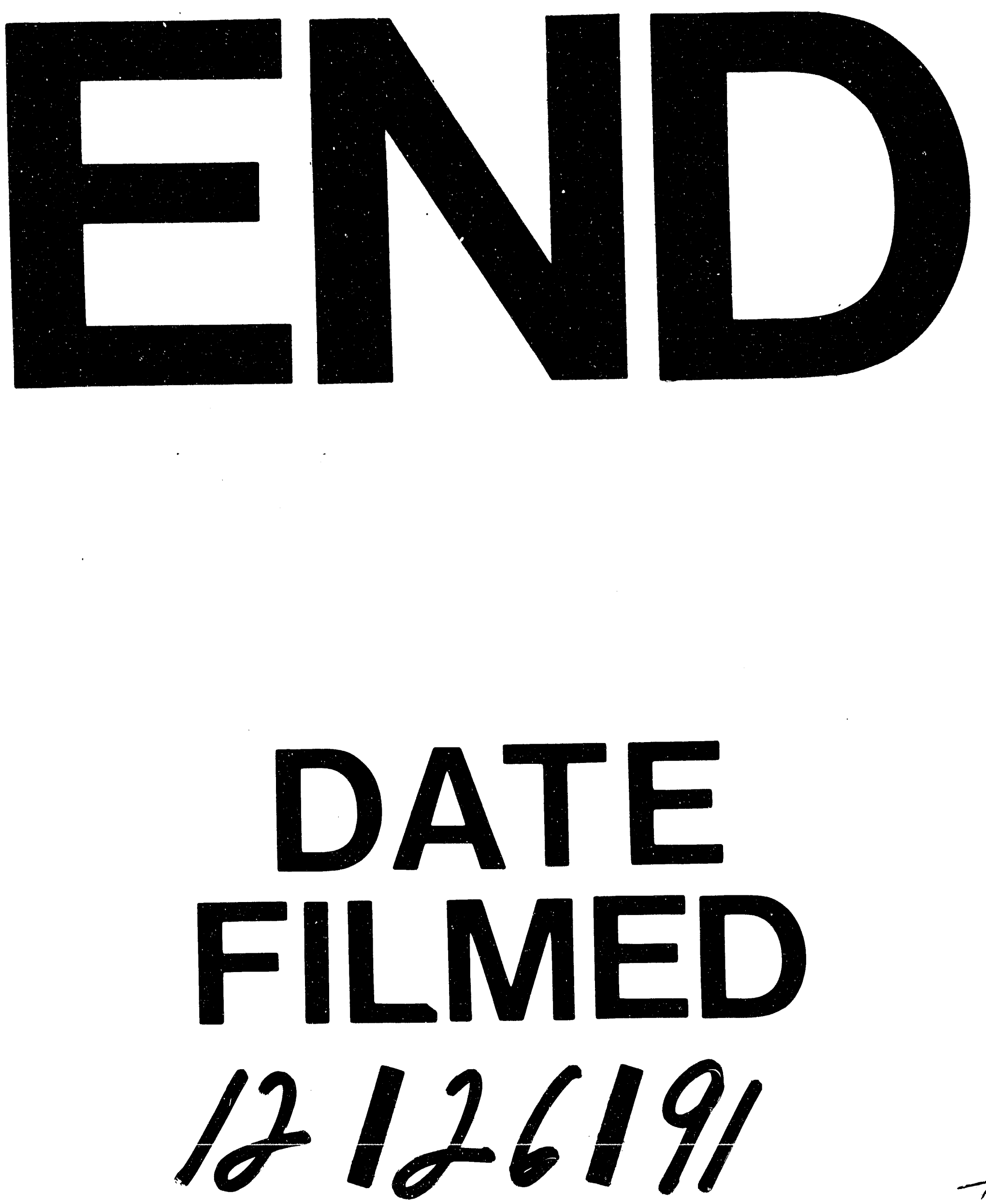
